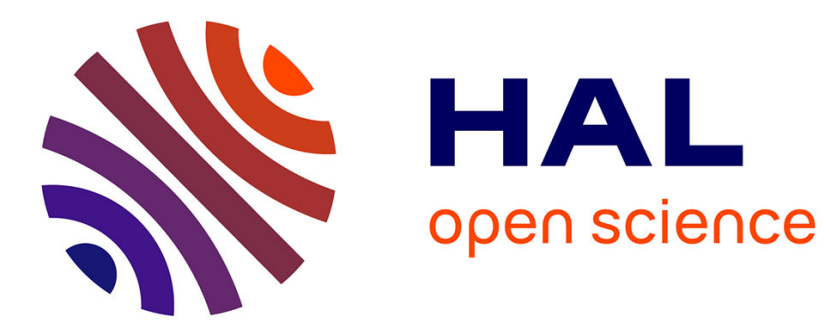

\title{
Odometers on Regular Languages
}

Valerie Berthe, Michel Rigo

\section{- To cite this version:}

Valerie Berthe, Michel Rigo. Odometers on Regular Languages. Theory of Computing Systems, 2007, 40, pp.001-031. 10.1007/s00224-005-1215-5 . lirmm-00130835

\section{HAL Id: lirmm-00130835 https://hal-lirmm.ccsd.cnrs.fr/lirmm-00130835}

Submitted on 27 Feb 2007

HAL is a multi-disciplinary open access archive for the deposit and dissemination of scientific research documents, whether they are published or not. The documents may come from teaching and research institutions in France or abroad, or from public or private research centers.
L'archive ouverte pluridisciplinaire HAL, est destinée au dépôt et à la diffusion de documents scientifiques de niveau recherche, publiés ou non, émanant des établissements d'enseignement et de recherche français ou étrangers, des laboratoires publics ou privés. 


\title{
ODOMETERS ON REGULAR LANGUAGES
}

\author{
VALÉRIE BERTHÉ AND MICHEL RIGO†
}

\begin{abstract}
Odometers or "adding machines" are usually introduced in the context of positional numeration systems built on a strictly increasing sequence of integers. We generalize this notion to systems defined on an arbitrary infinite regular language. In this latter situation, if $(A,<)$ is a totally ordered alphabet, then enumerating the words of a regular language $L$ over $A$ with respect to the induced genealogical ordering gives a one-to-one correspondence between $\mathbb{N}$ and $L$. In this general setting, the odometer is not defined on a set of sequences of digits but on a set of pairs of sequences where the first (resp. the second) component of the pair is an infinite word over $A$ (resp. an infinite sequence of states of the minimal automaton of $L$ ). We study some properties of the odometer like continuity, injectivity, surjectivity, minimality,... We then study some particular cases: we show the equivalence of this new function with the classical odometer built upon a sequence of integers whenever the set of greedy representations of all the integers is a regular language; we also consider substitution numeration systems as well as the connection with $\beta$-numerations.
\end{abstract}

\section{INTRODUCTION}

To any infinite regular language $L$ over a totally ordered alphabet $(A,<)$, an abstract numeration system $S=(L, A,<)$ is associated in the following way [22]. Enumerating the words of $L$ by increasing genealogical order gives a one-to-one correspondence between $\mathbb{N}$ and $L$ : the non-negative integer $n$ being represented by the $(n+1)$-th word of the ordered language $L$. In particular, these systems generalize classical positional systems like the $k$-ary systems, the Fibonacci system or more generally the numeration systems built on a sequence of integers satisfying a linear recurrence relation whose characteristic polynomial is the minimal polynomial of a Pisot number [4].

In this framework of abstract numeration systems, the properties of the socalled $S$-recognizable sets of integers have been extensively studied (see for instance $[22,31,32])$. Moreover, these abstract systems have been extended to allow not only the representation of integers but also of real numbers [23]. In this latter situation, a real number is represented by an infinite word which is the limit of a converging sequence of words in $L$. Clearly, these systems lead to the generalization of various concepts related to the representation of integers like the automatic sequences or the summatory functions of additive functions [20,33].

In this paper, we want to define and study the properties of odometers (also called adding machines) in the framework of abstract numeration systems built on an infinite regular language. In [19] odometers for positional numeration systems defined on a strictly increasing sequence $\left(U_{n}\right)_{n \in \mathbb{N}}$ of integers such that $U_{0}=1$ are investigated. In this latter situation, the odometer function is defined on the set $\mathcal{R}$ of right infinite words $\alpha_{0} \alpha_{1} \alpha_{2} \ldots$ satisfying a greedy property [14], i.e., for all $\ell \geq 0$,

$$
\sum_{i=0}^{\ell} \alpha_{i} U_{i}<U_{\ell+1}
$$

†Corresponding author. 
(We will also consider the greedy property (1) for finite words in the following.) The least significant digits are written first: the prefix $\alpha_{0} \cdots \alpha_{k}$ of a word in $\mathcal{R}$ has value $\alpha_{0} U_{0}+\cdots+\alpha_{k} U_{k}$. The odometer is thus defined as the infinite extension of the successor function acting on the finite words of the form $\alpha_{0} \cdots \alpha_{k}$. As an example, let us consider a finite word: the usual decimal representation of one hundred seventy-two is the word "172"; but here, we will write representations backward and so this number is written " 271 " and adding one leads to the word " 371 ". Moreover, the language of the finite words representing all the integers can be embedded into $\mathcal{R}$ by concatenating $0^{\omega}$ to the right of a greedy representation starting with the least significant digit first. So, one hundred seventy-two gives the element $271(0)^{\omega} \in \mathcal{R}$. Adding one to an infinite word in $\mathcal{R}$ can produce a carry propagating to the right. As an example, the application of the odometer to $9992(0)^{\omega}$ gives $0003(0)^{\omega}$. In the case of the Fibonacci system where $U_{0}=1, U_{1}=2$ and $U_{n+2}=U_{n+1}+U_{n}$ applying the odometer to 010101(0) ${ }^{\omega}$ gives 0000001(0) ${ }^{\omega}$ (indeed, to be in $\mathcal{R}$ the greedy condition (1), i.e., the pattern "11" does not occur, must be satisfied). For these reasons, we will consider mirror of representations all along this paper.

There is an important literature devoted to the study of odometers. Let us briefly quote [5] which continues the study of [19] from a combinatorial and topological point of view, and [6] for a metrical approach. Odometers can also be defined for two-sided dynamical systems as investigated in [15, 35], we refer to [37] for the golden ratio case. See also [7] for an ergodic application of this notion in the framework of unimodal maps and wild attractors. Lastly, let us mention [16] which studies the sequential properties of the successor function for positional numeration systems.

This paper is organized as follows. After recalling the basic notions required in this paper, we define in Section 2 the set $\widetilde{\mathcal{K}}$ on which the odometer acts, and state a few preliminary properties. Special focus is given on its extremal elements in Section 3, which allows us to define the odometer in Section 4. Its first properties (continuity, injectivity, surjectivity and minimality) are then stated in Section 5. We illustrate this study by making explicit the connection with a few wellknown situations where the odometer is perfectly described: we consider the case of positional number systems in Section 6, the case of substitution numeration systems (with special focus on Pisot substitutions) in Section 7, and the case of $\beta$-numeration in Section 8. We consider the possibility of getting a real representation of the odometer in Section 9 and end this paper by considering some special cases in Section 10.

\section{Preliminaries}

Let $A=\left\{a_{0}<a_{1}<\cdots<a_{k}\right\}$ be a finite and totally ordered alphabet. In this paper $L \subset A^{*}$ will always denote a regular language such that $a_{0}^{*} L \subseteq L$. In other words, $L$ is infinite and has the following property

$$
w \in L \Leftrightarrow \forall n \geq 0, a_{0}^{n} w \in L .
$$

In some sense, property (2) can be related to the property of numeration systems built on a sequence of integers $\left(U_{n}\right)_{n \in \mathbb{N}}$ such that if $w=w_{0} \cdots w_{k}$ is the greedy representation of an integer $w_{0} U_{0}+\cdots+w_{k} U_{k}$ (remember that we have taken the convention to write the least significant digit first) then $w 0^{n}, n \in \mathbb{N}$, still satisfies the greedy condition (1) and represents the same integer. Here, since we will consider the mirror of words in $L$, we will be able to write an arbitrary number of $a_{0}$ 's on the right of the mirror of any word in $L$ and still obtain mirror of words belonging to $L$. Property (2) will therefore ensure the embedding of the finite words 
of $L$ representing the non-negative integers into some set of infinite sequences that will be precised later (Definition 1 below).

The minimal automaton of $L$ is denoted $\mathcal{M}_{L}=\left(Q, q_{0}, A, \delta, F\right)$ where $Q$ is the set of states, $q_{0}$ is the initial state, $F \subseteq Q$ is the set of final states and $\delta: Q \times A \rightarrow Q$ is the transition function. We assume that $\delta$ is total or equivalently that $\mathcal{M}_{L}$ is complete, i.e., $\delta$ is defined for all pairs $(q, a) \in Q \times A$ (notice that even with this assumption, $\mathcal{M}_{L}$ might contain a sink, i.e., a non-final state $s$ such that for any $a \in A, \delta(s, a)=s)$. As usual, $\delta$ can be extended to $Q \times A^{*}$. As a consequence of property $(2), \mathcal{M}_{L}$ has a loop in $q_{0}$ of label $a_{0}$. For the properties of the minimal automaton, see for instance [12].

For any state $q \in Q$, we denote by $L_{q}$ the regular language accepted by $\mathcal{M}_{L}$ from state $q$,

$$
L_{q}=\left\{w \in A^{*} \mid \delta(q, w) \in F\right\}
$$

and by $\mathbf{u}_{q}(n)$ the number of words of length $n$ in $L_{q}$. In particular, $L=L_{q_{0}}$.

Since $A$ is totally ordered, we can order the words of $A^{*}$ using the genealogical ordering. Let $u, v \in A^{*}$. We say that $u<v$ if $|u|<|v|$ or if $|u|=|v|$ and there exist $p, u^{\prime}, v^{\prime} \in A^{*}, a, b \in A, a<b$ such that $u=p a u^{\prime}$ and $v=p b v^{\prime}$. If $M$ is a language over $A$, we define $\operatorname{Max}(M)$ as the set of the greatest words of each length in $M$, i.e.,

$$
\operatorname{Max}(M)=\{u \in M|\forall v \in M,| u|=| v \mid \Rightarrow v \leq u\} .
$$

Observe that for all $n \geq 0$, \# $\left(\operatorname{Max}(M) \cap A^{n}\right) \in\{0,1\}$. In the same way, we can also define the set $\operatorname{Min}(M)$ containing the smallest word of each length in $M$. It is well-known that if $M$ is regular then $\operatorname{Max}(M)$ and $\operatorname{Min}(M)$ are also regular [36].

If $w=w_{0} \cdots w_{\ell}$ is a word over $A$ then the reversal (or mirror) of $w$ is $w_{\ell} \cdots w_{0}$ and is denoted $\widetilde{w}$. If $M$ is a language, then $\widetilde{M}$ is the language $\{\widetilde{w} \mid w \in M\}$. We also consider the non-deterministic finite automaton $\widetilde{\mathcal{M}_{L}}=\left(Q, F, A, \widetilde{\delta},\left\{q_{0}\right\}\right)$ having the same set of states as $\mathcal{M}_{L}, F$ as set of initial states, the transition relation $\widetilde{\delta} \subseteq Q \times A \times Q$ is defined by

$$
(q, a, r) \in \widetilde{\delta} \Leftrightarrow \delta(r, a)=q .
$$

So, since $q_{0}$ is the only final state then a word $w$ is accepted by $\widetilde{\mathcal{M}}_{L}$ if and only if $\widetilde{w} \in L$. Since $\mathcal{M}_{L}$ is accessible, in $\widetilde{\mathcal{M}_{L}}$ for any state $q$ there exists at least one path from $q$ to $q_{0}$. Moreover, we also have a loop in $q_{0}$ of label $a_{0}$. Let us already observe that in our later developments, the set of final states of $\widetilde{\mathcal{M}}_{L}$ will be irrelevant since we mainly work with limits of words recognized by $\widetilde{\mathcal{M}_{L}}$.

We denote by $\widetilde{\mathcal{L}}$ the set of infinite words over $A$ which are the limits of the converging sequences of words belonging to $\widetilde{L}$. Otherwise stated, $x=x_{0} x_{1} x_{2} \ldots$ belongs to $\widetilde{\mathcal{L}}$ if there exists a sequence $\left(w_{n}\right)_{n \in \mathbb{N}}$ of words in $L$ such that for all $\ell>0$ there exist $N_{\ell}>0$ such that for all $n \geq N_{\ell}, \widetilde{w_{n}}$ and $x$ have a common prefix of length at least $\ell$. We use the topology induced by the infinite product topology on $A^{\mathbb{N}}$. Notice that if all the sates in $\mathcal{M}_{L}$ are final, then $\widetilde{\mathcal{L}}$ is the set of labels of infinite paths in $\widehat{\mathcal{M}_{L}}$.

Due to the non-deterministic behavior of $\widetilde{\mathcal{M}_{L}}$, the reading of a word in this automaton can lead to more than one path. As an example, assume that in $\mathcal{M}_{L}$ we have three states $p, q$ and $r$ such that $\delta(p, a)=r$ and $\delta(q, a)=r$ then in $\widetilde{\mathcal{M}}_{L}$ from state $r$ when reading $a$ both states $p$ and $q$ could be reached and a nondeterministic choice has to be made. Therefore, we will not only consider words but also the extra information given by the sequence of reached states. This is the reason of the introduction of the set $\widetilde{\mathcal{K}}$ defined below.

Definition 1. We define the set $\widetilde{\mathcal{K}} \subseteq(A \times Q)^{\omega}$ by $(x, y)=\left(x_{0} x_{1} x_{2} \ldots, y_{0} y_{1} y_{2} \ldots\right)$ belongs to $\widetilde{\mathcal{K}}$ if and only if the following conditions hold 
(1) $x$ belongs to $\widetilde{\mathcal{L}}$,

(2) $y_{0}$ belongs to $F$,

(3) for all $i \geq 0,\left(y_{i}, x_{i}, y_{i+1}\right)$ belongs to $\widetilde{\delta}$, i.e., $\delta\left(y_{i+1}, x_{i}\right)=y_{i}$.

Lemma 2. If $(x, y)=\left(x_{0} x_{1} x_{2} \ldots, y_{0} y_{1} y_{2} \ldots\right)$ belongs to $\widetilde{\mathcal{K}}$ then for all $k \in \mathbb{N}$, $x_{k} \cdots x_{0}$ belongs to $L_{y_{k+1}}$.

Proof. By definition of $\widetilde{\mathcal{K}}$, for $i=0, \ldots, k, \delta\left(y_{i+1}, x_{i}\right)=y_{i}$. Therefore in $\mathcal{M}_{L}$ we have a path from $y_{k+1}$ to $y_{0}$ of label $x_{k} \cdots x_{0}$. Since $y_{0}$ is a final state, this means that $x_{k} \cdots x_{0}$ is accepted from $y_{k+1}$.

Remark 3. Let $k \geq 0$. If $(x, y)=\left(x_{0} x_{1} x_{2} \ldots, y_{0} y_{1} y_{2} \ldots\right)$ belongs to $\widetilde{\mathcal{K}}$ then $y_{0} \cdots y_{k}$ is completely determined by $x_{0} \cdots x_{k}$ and $y_{k+1}$. This is due to the third condition in the definition of $\widetilde{\mathcal{K}}$ and because $\mathcal{M}_{L}$ is deterministic.

Definition 4. Let $j \geq 0$. A finite word $(x, y)=\left(x_{0} x_{1} \cdots x_{k}, y_{0} y_{1} \cdots y_{k}\right) \in$ $(A \times Q)^{k+1}, k>j,\left(\right.$ resp. an infinite word $\left.(x, y)=\left(x_{0} x_{1} \ldots, y_{0} y_{1} \ldots\right) \in(A \times Q)^{\omega}\right)$ is said to have the property $\max _{j}$ and we write $(x, y) \in \max _{j}$ if $x_{j} \cdots x_{0}$ belongs to $\operatorname{Max}\left(L_{y_{j+1}}\right)$. In the same way, $(x, y)$ has the property $\min _{j}$ if $x_{j} \cdots x_{0} \in$ $\operatorname{Min}\left(L_{y_{j+1}}\right)$.

Lemma 5. Let $(x, y)=\left(x_{0} x_{1} x_{2} \ldots, y_{0} y_{1} y_{2} \ldots\right) \in \widetilde{\mathcal{K}}$ and $j \geq 0$. If $(x, y)$ has property $\max _{j}\left(\right.$ resp. $\left.\mathbf{m i n}_{j}\right)$ then for all $k<j,(x, y)$ has also the property $\mathbf{m a x}_{k}$ (resp. $\left.\min _{k}\right)$.

Proof. Assume that $(x, y) \in \max _{j}$ but $(x, y) \notin \max _{\mathbf{k}}, k<j$. Therefore there exists $x_{k}^{\prime} \cdots x_{0}^{\prime}$ accepted from $y_{k+1}$ and genealogically greater than $x_{k} \cdots x_{0}$. So $x_{j} \cdots x_{k+1} x_{k}^{\prime} \cdots x_{0}^{\prime}$ belongs to $L_{y_{j+1}}$ and is greater than $x_{j} \cdots x_{0}$. This is a contradiction.

Corollary 6. Let $(x, y)=\left(x_{0} x_{1} x_{2} \ldots, y_{0} y_{1} y_{2} \ldots\right) \in \widetilde{\mathcal{K}}$ and $j \geq 0$. If $(x, y) \notin \max _{j}$ (resp. $\left.(x, y) \notin \min _{j}\right)$ then for all $i \geq j,(x, y) \notin \max _{i}\left(\operatorname{resp.}(x, y) \notin \mathbf{m i n}_{i}\right)$.

Let us now present some other properties of this set $\widetilde{\mathcal{K}}$.

Proposition 7. For each $x \in \widetilde{\mathcal{L}}$, there exists $y \in Q^{\omega}$ such that $(x, y)$ belongs to $\widetilde{\mathcal{K}}$.

Proof. If $w=w_{1} \cdots w_{\ell}$ is a word in $L$, we denote by $p(w)$ the word

$$
p(w):=\delta\left(q_{0}, w_{1}\right) \delta\left(q_{0}, w_{1} w_{2}\right) \cdots \delta\left(q_{0}, w_{1} \cdots w_{\ell}\right) \in Q^{\ell}
$$

which represents the path given by the states reached consecutively in $\mathcal{M}_{L}$ by reading $w$. Since $x$ belongs to $\widetilde{\mathcal{L}}$, there exists a sequence $\left(x_{n}\right)_{n \in \mathbb{N}}$ of words in $\widetilde{L}$ converging to $x$. For an infinite number of $n \in \mathbb{N}$, the last element of $p\left(\widetilde{x_{n}}\right)$ is a same state in $F$. We take the corresponding subsequence $\left(x_{k_{1}(n)}\right)_{n \in \mathbb{N}}$. For an infinite number of $n$, the words $p\left(\widetilde{x_{k_{1}(n)}}\right)$ have the same suffix of length two. So we consider the corresponding subsequence $\left(x_{k_{2}(n)}\right)_{n \in \mathbb{N}}$. If we iterate this process, $\left(x_{k_{n}(1)}\right)_{n \in \mathbb{N}}$ is converging to $x$ if $n$ tends to infinity and the reversal of the $p\left(\widetilde{x_{k_{n}(1)}}\right)$ 's are converging to an infinite word $y$ in $Q^{\omega}$ such that $(x, y)$ belongs to $\widetilde{\mathcal{K}}$.

Example 8. In this example, we consider a regular language $L \subset\{a<b<c\}^{*}$ satisfying the hypothesis $a^{*} L \subset L$ and given by its minimal automaton depicted in Figure 1. We just present some elements belonging to $\widetilde{\mathcal{K}}$ :

$$
\begin{gathered}
\left.\left((b b a)^{\omega},(210)^{\omega}\right),(a)^{\omega}, 1(0)^{\omega}\right),\left(b(a)^{\omega}, 2(1)^{\omega}\right) \\
\left(b a(b b c)^{\omega}, 10(210102021)^{\omega}\right) \text { and }\left(b a(b b c)^{\omega}, 10(021210102)^{\omega}\right) .
\end{gathered}
$$




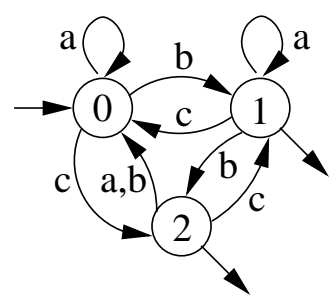

Figure 1. The minimal automaton of a language $L$.

As shown in the previous example, to one infinite word $x \in \widetilde{\mathcal{L}}$, it may correspond more than one sequence of states. If two such sequences give rise to elements in $\widetilde{\mathcal{K}}$ then they differ almost everywhere.

Lemma 9. Let $(x, y)$ and $\left(x, y^{\prime}\right)$ be two elements of $\widetilde{\mathcal{K}}$ such that $y \neq y^{\prime}$. Then there exists an index $i$ such that $y_{i} \neq y_{i}^{\prime}$ and for all $n \geq 0, y_{i+n} \neq y_{i+n}^{\prime}$.

Proof. This is a direct consequence of Remark 3.

The next proposition shows that to any finite word in $L$ corresponds at least one element in $\widetilde{\mathcal{K}}$. The same kind of properties holds in the case of numeration systems built on a sequence of integers. If $w$ is the greedy representation of an integer (least significant digit first), then $w(0)^{\omega}$ belongs to the set $\mathcal{R}$ of right infinite words satisfying the greedy property 1 .

Proposition 10. If $w=w_{k} \cdots w_{1}$ belongs to $L$ then there exists $y_{1} \cdots y_{k} \in Q^{k}$ such that $\left(w_{1} \cdots w_{k}\left(a_{0}\right)^{\omega}, y_{1} \cdots y_{k}\left(q_{0}\right)^{\omega}\right)$ belongs to $\widetilde{\mathcal{K}}$.

Proof. By our assumption (2) on $L$, if $w$ belongs to $L$ then $a_{0}^{n} w$ also belongs to $L, n \geq 0$. Using the same notation as in the proof of Proposition 7, if $y_{k} \cdots y_{1}=$ $p\left(w_{k} \cdots w_{1}\right)$ then $q_{0}^{n} y_{k} \cdots y_{1}=p\left(a_{0}^{n} w_{k} \cdots w_{1}\right)$. The result follows easily.

\section{Properties of $\operatorname{Max}(\widetilde{\mathcal{K}})$ and $\operatorname{Min}(\widetilde{\mathcal{K}})$}

For odometers defined upon classical positional systems related to a sequence $\left(U_{n}\right)_{n \in \mathbb{N}}$ of integers, some sequences of digits play a special role. Namely, they are the sequences for which the carry when adding one can propagate to infinity. A sequence $\alpha_{0} \alpha_{1} \alpha_{2} \ldots$ is of this kind if

$$
\sum_{i=0}^{\ell_{j}} \alpha_{i} U_{i}=U_{\ell_{j}+1}-1
$$

for a strictly increasing infinite sequence $\left(\ell_{j}\right)_{j \in \mathbb{N}}$ of indices. In our framework, the corresponding elements in $\widetilde{\mathcal{K}}$ will be defined as the elements in $\operatorname{Max}(\widetilde{\mathcal{K}})$. The elements which have the dual property will belong to $\operatorname{Min}(\widetilde{\mathcal{K}})$.

Definition 11. Let us define two particular subsets of $\widetilde{\mathcal{K}}$,

$$
\operatorname{Max}(\widetilde{\mathcal{K}})=\left\{(x, y) \in \widetilde{\mathcal{K}} \mid \forall i \geq 0,(x, y) \in \max _{i}\right\}
$$

and

$$
\operatorname{Min}(\widetilde{\mathcal{K}})=\left\{(x, y) \in \widetilde{\mathcal{K}} \mid \forall i \geq 0,(x, y) \in \min _{i}\right\} .
$$

Let us observe that following Lemma 5 , then it is sufficient in the definition of $\operatorname{Max}(\widetilde{\mathcal{K}})(\operatorname{resp} . \operatorname{Min} \widetilde{\mathcal{K}}))$ that there exist infinitely many $i$ such that $(x, y) \in \max _{i}$ (resp. $\left.(x, y) \in \mathbf{m i n}_{i}\right)$. In this section, we concentrate on the structural properties of those sets $\operatorname{Max}(\widetilde{\mathcal{K}})$ and $\operatorname{Min}(\widetilde{\mathcal{K}})$. The following lemma is obvious. 
Lemma 12. Let $L$ be a regular language satisfying our assumption (2).

- A word $w$ belongs to $\operatorname{Min}(L)$ if and only if for all $n \geq 0, a_{0}^{n} w$ belongs to $\operatorname{Min}(L)$ (assuming that $a_{0}$ is the smallest letter in the ordered alphabet $A$ ).

- Let $q$ be a state of $\mathcal{M}_{L}$. If vw belongs to $\operatorname{Max}\left(L_{q}\right)$ then the word $w$ belongs to $\operatorname{Max}\left(L_{\delta(q, v)}\right)$.

Definition 13. Let $w$ be the smallest word in $\operatorname{Min}(L)$, i.e., $w$ is the first word in the ordered language $L$. If $w=w_{1} \cdots w_{\ell}$ is not the empty word (i.e., if $q_{0} \notin F$ ) then we have a path in $\mathcal{M}_{L}$ of the form

$$
q_{0} \stackrel{w_{1}}{\longrightarrow} q_{1} \stackrel{w_{2}}{\longrightarrow} \cdots \stackrel{w_{\ell}}{\longrightarrow} q_{\ell} \in F .
$$

We set $\mathbf{0}=\left(\widetilde{w}\left(a_{0}\right)^{\omega}, q_{\ell} \cdots q_{1}\left(q_{0}\right)^{\omega}\right)$. Otherwise $w=\varepsilon$ and we set $\mathbf{0}=\left(\left(a_{0}\right)^{\omega},\left(q_{0}\right)^{\omega}\right)$.

Proposition 14. The sets $\operatorname{Max}(\widetilde{\mathcal{K}})$ and $\operatorname{Min}(\widetilde{\mathcal{K}})$ are non-empty.

Proof. As a consequence of Lemma 5 and Lemma 12, the element 0 given in Definition 13 belongs to $\operatorname{Min}(\widetilde{\mathcal{K}})$.

We use the same idea as in the proof of Proposition 7. Let $w_{i}$ be the $i$-th word of $\operatorname{Max}(L)$ (clearly, $\left|w_{i}\right|<\left|w_{i+1}\right|$ for all $i \geq 1$ ). An infinite number of $w_{i}$ 's have the same last letter $a_{k_{1}}$ and lead in $\mathcal{M}_{L}$ from $q_{0}$ to a same final state $q_{k_{1}}$. We therefore consider the corresponding subsequence $\left(w_{k_{1}(n)}\right)_{n \in \mathbb{N}}$ built upon those $w_{i}$ 's. We iterate this process: an infinite number of words among the $w_{k_{1}(n)}$ 's have the same suffix $a_{k_{2}} a_{k_{1}}$ and finally lead in $\mathcal{M}_{L}$ to the states $q_{k_{2}}$ followed by $q_{k_{1}}$. Therefore we build a sequence converging to

$$
\left(a_{k_{1}} a_{k_{2}} \cdots, q_{k_{1}} q_{k_{2}} \cdots\right) \text {. }
$$

Thanks to Lemma 12 , this element belongs to $\operatorname{Max}(\widetilde{\mathcal{K}})$.

Example 15. We consider the language and the automaton given in Example 8. It is easy to check that $\left(b(c)^{\omega}, 2(120)^{\omega}\right),\left(c^{\omega},(120)^{\omega}\right)$ and $\left(c^{\omega},(201)^{\omega}\right)$ belong to $\operatorname{Max}(\widetilde{\mathcal{K}})$. We also have $\mathbf{0}=\left(b(a)^{\omega}, 1(0)^{\omega}\right)$ and $\left(a^{\omega}, 1^{\omega}\right)$ as elements of $\operatorname{Min}(\widetilde{\mathcal{K}})$. To show that these elements are the only ones, we will need some more results about the structure of $\operatorname{Max}\left(L_{q}\right)$ and $\operatorname{Min}\left(L_{q}\right)$.

In some particular cases, the structure of $\operatorname{Max}\left(L_{q}\right)$ is easy to obtain.

Notation 16. Let $q$ be a state in $\mathcal{M}_{L}$. If there exists $a \in A$ such that $\delta(q, a)$ is not the sink then we denote by $m(q)$ the largest letter having this property, otherwise we set $m(q)=\varepsilon$.

Recall that a state $s$ is a sink if for any $a \in A, \delta(s, a)=s$ and $s$ is not a final state. Let us introduce a small algorithm to detect what we will call the maximal cycles in $\mathcal{M}_{L}$.

Algorithm 17. Let $q \in Q$.

- Set $y_{0} \leftarrow q$ and $i \leftarrow 0$.

- If $m\left(y_{i}\right) \neq \varepsilon$ then set $y_{i+1} \leftarrow \delta\left(y_{i}, m\left(y_{i}\right)\right)$ and $i \leftarrow i+1$. Otherwise stop the algorithm.

- If $y_{0}, \ldots, y_{i}$ are all different, repeat the previous step. Otherwise, a cycle is found and stop the algorithm.

After applying this algorithm to a state $q \in Q$ which is not the sink, we can have two kinds of situations. If we encounter some state $y_{k}$ such that $m\left(y_{k}\right)=\varepsilon$ then we have obtained something like

$$
y_{0} \stackrel{m\left(y_{0}\right)}{\longrightarrow} y_{1} \stackrel{m\left(y_{1}\right)}{\longrightarrow} \cdots \longrightarrow y_{k-1} \stackrel{m\left(y_{k-1}\right)}{\longrightarrow} y_{k}
$$


where all the $y_{i}$ 's are different and $y_{k}$ belongs necessarily to $F$ (because $\mathcal{M}_{L}$ is minimal). Or we have the situation

$$
y_{0} \stackrel{m\left(y_{0}\right)}{\longrightarrow} y_{1} \stackrel{m\left(y_{1}\right)}{\longrightarrow} \cdots \longrightarrow \mathbf{y}_{\mathbf{k}} \stackrel{m\left(y_{k}\right)}{\longrightarrow} \cdots \longrightarrow y_{k+n} \stackrel{m\left(y_{k}+n\right)}{\longrightarrow} \mathbf{y}_{\mathbf{k}}
$$

where $y_{0}, \ldots, y_{k+n}$ are all different; we say that $\left(y_{k}, m\left(y_{k}\right), \ldots, y_{k+n}, m\left(y_{k+n}\right), y_{k}\right)$ is a maximal cycle starting in $y_{k}$ and the word $m\left(y_{k}\right) \cdots m\left(y_{k+n}\right)$ is the label of this cycle. Notice that two maximal cycles have no state in common or share exactly the same states. In this latter case, the label of one of the two cycles is a cyclic permutation of the other one.

Example 18. Considering the automaton of Example 8, we have three maximal cycles: $(0, c, 2, c, 1, c, 0),(2, c, 1, c, 0, c, 2)$ and $(1, c, 0, c, 2, c, 1)$ all having the same label $c c c$ and sharing the same states.

Lemma 19. If $\mathcal{C}$ is a maximal cycle of label $w$ starting in $q$, then there exist an integer $k \leq|w|$ depending only on $\mathcal{C}$ and $k$ words $u_{1}, \ldots, u_{k}$ of minimal length such that $\left|u_{i}\right| \not \equiv\left|u_{j}\right| \bmod |w|$ if $i \neq j$ and

$$
\operatorname{Max}\left(L_{q}\right)=w^{*}\left\{u_{1}, \ldots, u_{k}\right\} .
$$

Proof. Let $w$ be the label of a maximal cycle $\mathcal{C}$ starting in $q$. If $v$ belongs to $\operatorname{Max}\left(L_{q}\right)$ then by construction of the maximal cycle, $w v$ also belongs to $\operatorname{Max}\left(L_{q}\right)$. Assume now that $u, v \in \operatorname{Max}\left(L_{q}\right)$ are such that $|u| \equiv|v| \bmod |w|$ and $|u|<|v|$. Therefore, there exists $i$ such that $w^{i} u$ belongs to $\operatorname{Max}\left(L_{q}\right)$ and $\left|w^{i} u\right|=|v|$. But $\operatorname{Max}\left(L_{q}\right)$ contains at most one word of each length, so $w^{i} u=v$. Consequently, if $v$ belongs to $\operatorname{Max}\left(L_{q}\right)$ then it is of the form $w^{n} u$ for some $n \geq 0$ and $w$ is not a prefix of $u$. For each $j \in\{0, \ldots, k-1\}$ there is at most one $u$ of this kind such that $|u| \equiv j$ $\bmod |w|$ (actually $u$ is the smallest word of length $j+n|w|$ possibly belonging to $\left.\operatorname{Max}\left(L_{q}\right), n \geq 0\right)$. Notice that it does not mean that $|u|<|w|$. Clearly two states in the same maximal cycle give rise to the same kind of maximal set.

It is more difficult to express the form of $\operatorname{Max}\left(L_{q}\right)$ when this set is infinite and $q$ does not belong to a maximal cycle. But hopefully we have a more general result extending Lemma 19 which holds even if $q$ does not belong to a maximal cycle. Indeed, since \# $\left(\operatorname{Max}\left(L_{q}\right) \cap A^{n}\right) \leq 1$ for all $n \in \mathbb{N}$ then it is well-known (see [27] or [36]) that there exists a finite set $R$ of words, an integer $k \geq 0$ and words $u_{i}, w_{i} \in A^{*}, v_{i} \in A^{+}, i=0, \ldots, k$ such that

$$
\operatorname{Max}\left(L_{q}\right)=\bigcup_{i=0}^{k} u_{i} v_{i}^{*} w_{i} \cup R
$$

where the languages $u_{i} v_{i}^{*} w_{i}$ are pairwise disjoint and also disjoint from $R$. Otherwise stated, if $i \neq j$ then

$$
\left\{\left|u_{i} w_{i}\right|+n\left|v_{i}\right|: n \in \mathbb{N}\right\} \cap\left\{\left|u_{j} w_{j}\right|+n\left|v_{j}\right|: n \in \mathbb{N}\right\}=\emptyset
$$

and $\left\{\left|u_{i} w_{i}\right|+n\left|v_{i}\right|: n \in \mathbb{N}\right\} \cap|R|=\emptyset$, for all $i(|R|$ denotes the set of lengths of elements of $R$ ). One can observe that the form of $\operatorname{Max}\left(L_{q}\right)$ given in Lemma 19 is a special case of (3).

Proposition 20. Any element in $\operatorname{Max}(\widetilde{\mathcal{K}})$ is ultimately periodic and $\operatorname{Max}(\widetilde{\mathcal{K}})$ is finite.

Proof. (a) The ideas of the first part of this proof are the same as in [23, Lemma 7]. Let $q$ be such that $\# \operatorname{Max}\left(L_{q}\right)=\infty$. If $x$ is a word in $\operatorname{Max}\left(L_{q}\right)$ of length large enough then thanks to (3) there exist unique words $u, v, w$ (depending on $x$ ) such that $x=u v^{n} w$. Among

$$
\delta(q, u), \delta(q, u v), \ldots, \delta\left(q, u v^{\# Q}\right)
$$


a same state appears at least twice. Let $t$ be the first state appearing twice in this list. Let $i<j$ be the smallest integers such that $\delta\left(q, u v^{i}\right)=\delta\left(q, u v^{j}\right)=t$. We set $P=(j-i)|v|$. We can already notice that $P$ is bounded by $\# Q \cdot|v|$. In what follows, we write simply $t, i, j, P$ assuming that the word $x$ is understood from the context.

(b) If $z=z_{0} \cdots z_{\ell}$ is a word over $A$ and $f$ is a state, we build the pair

$$
p_{f}(z):=\left(z_{0} \cdots z_{\ell}, \delta\left(f, z_{0}\right) \delta\left(f, z_{0} z_{1}\right) \cdots \delta\left(f, z_{0} \cdots z_{\ell}\right)\right) .
$$

Actually, $p_{f}(z)$ contains a word $z$ and the sequence of states reached from $f$ when reading $z$ in $\mathcal{M}_{L}$ (since $\mathcal{M}_{L}$ is complete, $\delta\left(f, z_{0} \cdots z_{k}\right)$ is defined for $0 \leq k \leq \ell$ ). Consider again the word $x=u v^{n} w \in \operatorname{Max}\left(L_{q}\right)$ introduced in (a). For $n$ large enough, $p_{q}\left(u v^{n} w\right)$ is a word over $A \times Q$ having

i) a non-periodic prefix $p_{q}\left(u v^{i}\right)$ of length bounded by $|u|+\# Q \cdot|v|$;

ii) a maximal periodic factor having a period of length $P$; actually the Euclidean division of $n-i$ by $P /|v|$ gives

$$
n-i=m \frac{P}{|v|}+r \quad \text { with } r<P /|v| \text {. }
$$

The periodic factor corresponding to $v^{m P /|v|}$ is $p_{t}\left(v^{m P /|v|}\right)$ and the period corresponding to $v^{P /|v|}$ is $p_{t}\left(v^{P /|v|}\right)$ where $t$ is as in (a) the first state appearing twice in the list (4);

iii) a non-periodic suffix of length bounded by $|w|+P$, indeed this factor corresponds to $v^{r} w$ and is of the form $p_{t}\left(v^{r} w\right)$.

For a better understanding, the situation is sketched in Figure 2.

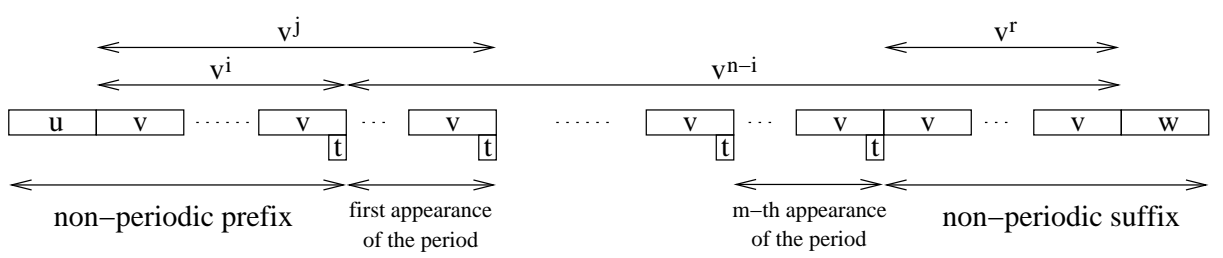

FIGURE 2. A schematic representation of $p_{q}\left(u v^{n} w\right)$.

(c) Let $n^{\prime}>n$ and $x^{\prime}=u v^{n^{\prime}} w$. Then $p_{q}\left(u v^{n} w\right)$ and $p_{q}\left(u v^{n^{\prime}} w\right)$ have the same prefix corresponding to $u v^{i}$. The periodic factors have the same period of length $P$ but the number of repetitions could be larger for $x^{\prime}$. Finally, if $n$ and $n^{\prime}$ are not congruent modulo $P /|v|$ then the corresponding suffixes could be different, otherwise the suffixes are the same. Notice that there are only finitely many possible suffixes corresponding to the words of the form $v^{r} w$ for $r=0, \ldots, P /|v|-1$.

(d) From the previous observations, we can easily exhibit elements in $\operatorname{Max}(\widetilde{\mathcal{K}})$. Let $n_{0}$ be large enough and set $x_{m}=p_{q}\left(u v^{n_{0}+m P /|v|} w\right)$ for $m \geq 0$. From the previous point, $\widetilde{x_{m}}$ is converging to an ultimately periodic element in $\widetilde{\mathcal{K}}$. From Lemma 12 , this element belongs to $\operatorname{Max}(\widetilde{\mathcal{K}})$.

(e) Clearly, any element $(x, y)=\left(x_{0} x_{1} \ldots, y_{1} y_{2} \ldots\right)$ in $\operatorname{Max}(\widetilde{\mathcal{K}})$ is ultimately periodic. Since $Q$ is finite, a state $q$ must appear infinitely often in $y$ say in strictly increasing positions $k(n)$. For each $n, x_{k(n)} \cdots x_{0}$ belongs to $\operatorname{Max}\left(L_{q}\right)$ and the words of this kind have a longer and longer common suffix when $n$ is increasing. As a consequence of $(\mathrm{a}),(x, y)$ is ultimately periodic with $x$ of the form $\widetilde{w}(\widetilde{v})^{\omega}$, for some finite words $v$ and $w$.

(f) In (d), we have obtained elements of $\operatorname{Max}(\widetilde{\mathcal{K}})$ of a special form but in (e) we have shown that any element in $\operatorname{Max}(\widetilde{\mathcal{K}})$ is of this kind. To conclude, we have to 
show that $\operatorname{Max}(\widetilde{\mathcal{K}})$ is finite. First from (3), for each state $q$ the number of words $u_{i}$, $v_{i}, w_{i}$ used to obtain the structure of $\operatorname{Max}\left(L_{q}\right)$ is finite. For each of these 3 -tuples $\left(u_{i}, v_{i}, w_{i}\right)$ of words, we can obtain ultimately periodic elements in $\operatorname{Max}(\widetilde{\mathcal{K}})$ but the period of such an element is bounded by $\# Q \cdot\left|v_{i}\right|$ (see (a)) and the length of its prefix is bounded by $\left|w_{i}\right|+\# Q \cdot\left|v_{i}\right|$ (see (b)). In other words, we have a finite number of 3 -tuples $\left(u_{i}, v_{i}, w_{i}\right)$ each one giving at most a finite number of elements in $\operatorname{Max}(\widetilde{\mathcal{K}})$.

This proof shows that the elements of $\operatorname{Max}(\widetilde{\mathcal{K}})$ can be determined by the knowledge of the languages $\operatorname{Max}\left(L_{q}\right)$. As we will see in the following example, obtaining the decomposition of the form (3) for the languages $\operatorname{Max}\left(L_{q}\right)$ gives rise to all the elements in $\operatorname{Max}(\widetilde{\mathcal{K}})$. Moreover, observe that these languages $\operatorname{Max}\left(L_{q}\right)$ can be efficiently obtained from $\mathcal{M}_{L}$.

Naturally, Algorithm 17, Lemma 19 and Proposition 20 are easily adapted to the set $\operatorname{Min}(\widetilde{\mathcal{K}})$. In this case, similarly as in Notation 16, if there exists $a \in A$ such that $\delta(q, a)$ is not the sink then we denote by $m(q)$ the smallest letter having this property.

Example 21. Continuing again Example 8. We are now able to show that $\operatorname{Max}(\widetilde{\mathcal{K}})$ contains exactly the elements $\left(b(c)^{\omega}, 2(120)^{\omega}\right),\left(c^{\omega},(120)^{\omega}\right)$ and $\left(c^{\omega},(201)^{\omega}\right)$. We have a maximal cycle of label $c c c$ containing the three states of $\mathcal{M}_{L}$, so using Lemma 19 we obtain $\operatorname{Max}\left(L_{0}\right)=(c c c)^{*}\{c, c c, c c b\}, \operatorname{Max}\left(L_{1}\right)=(c c c)^{*}\{\varepsilon, b, c c\}$ and $\operatorname{Max}\left(L_{2}\right)=(c c c)^{*}\{\varepsilon, c, c b\}$. Let us first see which elements in $\operatorname{Max}(\widetilde{\mathcal{K}})$ come from the words in $\operatorname{Max}\left(L_{0}\right)$. The word $(c c c)^{n} c$ read from the state 0 gives in $\mathcal{M}_{L}$ the path

$$
0 \stackrel{c}{\rightarrow} 2 \stackrel{c}{\rightarrow} 1 \stackrel{c}{\rightarrow} 0 \cdots \stackrel{c}{\rightarrow} 2 \stackrel{c}{\rightarrow} 1 \stackrel{c}{\rightarrow} 0 \stackrel{c}{\rightarrow} 2 .
$$

With the notation of the proof of Proposition 20, we have

$$
p_{0}\left((c c c)^{n} c\right)=\left((c c c)^{n} c,(210)^{n} 2\right) .
$$

Reading this path from the right and letting $n$ tends to infinity gives the element $\left(c^{\omega}, 2(012)^{\omega}\right)=\left(c^{\omega},(201)^{\omega}\right)$. In the same way, the word $(c c c)^{n} c c$ gives $\left(c^{\omega},(120)^{\omega}\right)$ and finally $(c c c)^{n} c c b$ gives $\left(b(c)^{\omega}, 2(120)^{\omega}\right)$. If we do the same for the words in $\operatorname{Max}\left(L_{i}\right), i=1,2$, then we consider paths starting in $i$ and we obtain exactly the same three elements of $\widetilde{\mathcal{K}}$. It is clear that each set $\operatorname{Max}\left(L_{q}\right)$ produces the same elements of $\operatorname{Max}(\widetilde{\mathcal{K}})$ because all the states are in the same maximal cycle.

Let us now show that $\operatorname{Min}(\widetilde{\mathcal{K}})$ contains exactly $\mathbf{0}=\left(b(a)^{\omega}, 1(0)^{\omega}\right)$ and $\left(a^{\omega}, 1^{\omega}\right)$. Here we have two minimal cycles: $(0, a, 0)$ and $(1, a, 1)$. So thanks to the analogue of Lemma 19, we have $\operatorname{Min}\left(L_{0}\right)=a^{*} b$ and $\operatorname{Min}\left(L_{1}\right)=a^{*}$. From the analogue of (3), one finds $\operatorname{Min}\left(L_{2}\right)=a^{*} a b \cup\{\varepsilon, c\}$. For instance, starting in state 2 and reading $a^{n} a b$ gives the path

$$
2 \stackrel{a}{\rightarrow} 0 \stackrel{a}{\rightarrow} 0 \cdots \stackrel{a}{\rightarrow} 0 \stackrel{a}{\rightarrow} 0 \stackrel{b}{\rightarrow} 1
$$

Reading this path from the right and letting $n$ tends to infinity gives the element $\mathbf{0 .}$ Starting in 0 with $a^{n} b$ also leads to the same element $\mathbf{0}$. Finally starting in 1 with $a^{n}$ gives $\left(a^{\omega}, 1^{\omega}\right)$. Obviously, if two states $q$ and $q^{\prime}$ belong to two different minimal cycles then the sets $\operatorname{Min}\left(L_{q}\right)$ and $\operatorname{Min}\left(L_{q^{\prime}}\right)$ will never lead to a same element in $\operatorname{Min}(\widetilde{\mathcal{K}})$ because the two cycles have no state in common. 


\section{Defining THE ODOMETER}

In [19], if a sequence $\alpha_{0} \alpha_{1} \alpha_{2} \ldots$ of digits belonging to $\mathcal{R}$, i.e., satisfying the greedy condition (1), is such that there exists $M$ such that for all $\ell \geq M$

$$
\left[\alpha_{0} \cdots \alpha_{\ell}\right]:=\sum_{i=0}^{\ell} \alpha_{i} U_{i}<U_{\ell+1}-1
$$

then the odometer maps $\alpha_{0} \alpha_{1} \alpha_{2} \ldots$ onto $\alpha_{0}^{\prime} \cdots \alpha_{k}^{\prime} \alpha_{k+1} \alpha_{k+2} \ldots \in \mathcal{R}$ where $\alpha_{0}^{\prime} \cdots \alpha_{k}^{\prime}$ is the representation of $\left[\alpha_{0} \cdots \alpha_{k}\right]+1$ computed through the greedy algorithm (and it is shown that the result is independent of the choice of the index $k \geq M)$. Obviously, the representations of $\left[\alpha_{0} \cdots \alpha_{k}\right]$ and $\left[\alpha_{0} \cdots \alpha_{k}\right]+1$ have the same length. Otherwise, infinitely often the situation $\left[\alpha_{0} \cdots \alpha_{\ell}\right]=U_{\ell+1}-1$ occurs and then the odometer is defined to map $\alpha_{0} \alpha_{1} \alpha_{2} \ldots$ onto $0^{\omega}$.

Here we want to do the same in the context of abstract numeration systems and define a function $\tau_{L}: \widetilde{\mathcal{K}} \rightarrow \widetilde{\mathcal{K}}$, or simply $\tau$ if $L$ is clearly understood, having the adding behavior awaited for an odometer. First we define $\tau$ on $\widetilde{\mathcal{K}} \backslash \operatorname{Max}(\widetilde{\mathcal{K}})$. Assume that for $(x, y)=\left(x_{0} x_{1} \ldots, y_{0} y_{1} \ldots\right) \in \widetilde{\mathcal{K}}$ there exists $i \geq 0$ such that $(x, y) \notin \mathbf{m a x}_{i}$. For each state $q$ of $\mathcal{M}_{L}$, we define the function

$$
\operatorname{Succ}_{q}: L_{q} \rightarrow L_{q}
$$

mapping the $k$-th word in the genealogically ordered language $L_{q}$ to the $(k+1)$-th one in the same language (if $L_{q}$ is finite, we decide that $\operatorname{Succ}_{q}$ maps the largest word in $L_{q}$ onto the smallest one). Since $(x, y) \notin \mathbf{m a x}_{i}$, it is clear that $x_{i} \cdots x_{0}$ and $\operatorname{Succ}_{y_{i+1}}\left(x_{i} \cdots x_{0}\right)$ have the same length. Let us denote this latter word belonging to $L_{y_{i+1}}$ by $x_{i}^{\prime} \cdots x_{0}^{\prime}$. We set $y_{i}^{\prime}=\delta\left(y_{i+1}, x_{i}^{\prime}\right)$ and $y_{j}^{\prime}=\delta\left(y_{j+1}^{\prime}, x_{j}^{\prime}\right)$ for $j=i-1, \ldots, 0$. In other words, $y_{i}^{\prime}, \ldots, y_{0}^{\prime}$ are the states reached in $\mathcal{M}_{L}$ when reading $x_{i}^{\prime} \cdots x_{0}^{\prime}$ from $y_{i+1}$. In particular, observe that $y_{0}^{\prime}$ belongs to $F$. Hence $\left(x_{0}^{\prime} \cdots x_{i}^{\prime} x_{i+1} \ldots, y_{0}^{\prime} \cdots y_{i}^{\prime} y_{i+1} \ldots\right)$ belong to $\widetilde{\mathcal{K}}$. The function $\tau$ is defined by

$$
\tau\left(x_{0} \cdots x_{i} x_{i+1} \ldots, y_{0} \cdots y_{i} y_{i+1} \ldots\right)=\left(x_{0}^{\prime} \cdots x_{i}^{\prime} x_{i+1} \ldots, y_{0}^{\prime} \cdots y_{i}^{\prime} y_{i+1} \ldots\right) .
$$

We have to show that $\tau$ is well-defined. Assume that there exist $i<j$ such that $(x, y) \notin \max _{i}$ and $(x, y) \notin \mathbf{m a x}_{j}$. (Notice that in view of Corollary 6 , if $(x, y) \notin$ $\max _{i}$ then for all $k \geq i,(x, y) \notin \max _{k}$.) Then the previous construction does not depend on the choice of the index. Indeed, notice that by definition of $\widetilde{\mathcal{K}}$, $\delta\left(y_{j+1}, x_{j} \cdots x_{i+1}\right)=y_{i+1}$ and as a consequence of the genealogical ordering,

$$
\operatorname{Succ}_{y_{j+1}}\left(x_{j} \cdots x_{i+1} x_{i} \cdots x_{0}\right)=x_{j} \cdots x_{i+1} \operatorname{Succ}_{y_{i+1}}\left(x_{i} \cdots x_{0}\right) .
$$

Therefore, as a consequence of Remark 3, the corresponding sequences of states are the same: if $y_{j}^{\prime \prime} \cdots y_{0}^{\prime \prime}$ are the states reached in $\mathcal{M}_{L}$ when reading $\operatorname{Succ}_{y_{j+1}}\left(x_{j} \cdots x_{0}\right)$ from $y_{j+1}$, we have

$$
y_{j}^{\prime \prime} \cdots y_{i+1}^{\prime \prime} y_{i}^{\prime \prime} \cdots y_{0}^{\prime \prime}=y_{j} \cdots y_{i+1} y_{i}^{\prime} \cdots y_{0}^{\prime} .
$$

Thus, the value of $\tau$ does not depend on the index $i$ such that $(x, y) \notin \max _{i}$.

Example 22. We still consider the language and the automaton given in Example

8. For instance, $(x, y)=\left(\right.$ bbaccb $\left.(a)^{\omega}, 210201(0)^{\omega}\right)$ belongs to $\widetilde{\mathcal{K}}$. The word $b$ belongs to $\operatorname{Max}\left(L_{1}\right)$ so $(x, y) \in \max _{1}$ but $b b$ belongs to $L_{0} \backslash \operatorname{Max}\left(L_{0}\right)$ so $(x, y) \notin \max _{2}$. It is easy to see that the next word accepted from 0 is $c c$ and the path is $0 \stackrel{c}{\rightarrow} 2 \stackrel{c}{\rightarrow} 1$, thus

$$
\tau\left(b b\left|\operatorname{accb}(a)^{\omega}, 21\right| 0201(0)^{\omega}\right)=\left(c c\left|a c c b(a)^{\omega}, 12\right| 0201(0)^{\omega}\right) .
$$

If we had considered the word $c c a b b$ accepted from state 1 (because $(x, y) \notin \max _{5}$ ), the next word in $L_{1}$ is ccacc and this would have lead to the same element in $\widetilde{\mathcal{K}}$ :

$$
\tau\left(\text { bbacc }\left|b(a)^{\omega}, 21020\right| 1(0)^{\omega}\right)=\left(\operatorname{ccacc}\left|b(a)^{\omega}, 12020\right| 1(0)^{\omega}\right) .
$$


In the next section, we will see that in general, the continuity of the odometer cannot be ensured on $\operatorname{Max}(\widetilde{\mathcal{K}})$ whatever is the value taken by $\tau$ for the points in this set (see Example 29). Therefore, we decide that for all $(x, y) \in \operatorname{Max}(\widetilde{\mathcal{K}})$, $\tau(x, y)=\mathbf{0}$, where $\mathbf{0}$ is the canonical element of $\operatorname{Min}(\widetilde{\mathcal{K}})$ given in Definition 13.

Remark 23. We can as in [19] or [40] define a partial ordering on $\widetilde{\mathcal{K}}$, called antipodal order, in the following way. We have $(x, y) \prec\left(x^{\prime}, y^{\prime}\right)$ if $(x, y)=\left(x^{\prime}, y^{\prime}\right)$ or there exists some index $k$ such that $x_{k}<x_{k}^{\prime}$ and for all $j>k,\left(x_{j}, y_{j}\right)=\left(x_{j}^{\prime}, y_{j}^{\prime}\right)$. The elements in $\operatorname{Max}(\widetilde{\mathcal{K}})$ are therefore the maximal elements in $(\widetilde{\mathcal{K}}, \prec)$. For any $(x, y) \notin \operatorname{Max}(\widetilde{\mathcal{K}})$, then its image under $\tau$ is the smallest (with respect to $\prec$ ) of all the elements in $\widetilde{\mathcal{K}}$ which are larger than $(x, y)$. Hence the map $\tau$ is a successor function which can be considered as an adic transformation following [40].

\section{Properties of the odometers}

Proposition 24. The application $\tau$ is surjective onto $\widetilde{\mathcal{K}} \backslash \operatorname{Min}(\widetilde{\mathcal{K}})$.

Proof. The proof is immediate. Let $(x, y)=\left(x_{0} x_{1} \ldots, y_{0} y_{1} \ldots\right)$ be such that $(x, y)$ is not in $\min _{i}$ for some $i$. Therefore, there exists a word $x^{\prime}=x_{i}^{\prime} \cdots x_{0}^{\prime}$ of length $i+1$ such that $\operatorname{Succ}_{y_{i+1}}\left(x_{i}^{\prime} \cdots x_{0}^{\prime}\right)=x_{i} \cdots x_{0}$. As usual, if $y^{\prime}=y_{i}^{\prime} \cdots y_{0}^{\prime}$ is the path followed in $\mathcal{M}_{L}$ from $y_{i+1}$ when reading $x^{\prime}$ then

$$
\tau\left(\widetilde{x^{\prime}} x_{i+1} x_{i+2} \ldots, \widetilde{y^{\prime}} y_{i+1} y_{i+2} \ldots\right)=(x, y) .
$$

Remark 25. A similar result holds in the framework of positional number systems: the odometer is proved to be surjective if and only if $0^{\omega}$ admits an antecedent (see [19]).

Proposition 26. The application $\tau$ is injective on $\widetilde{\mathcal{K}} \backslash \operatorname{Max}(\widetilde{\mathcal{K}})$.

Proof. Let $(x, y)=\left(x_{0} x_{1} \ldots, y_{0} y_{1} \ldots\right)$ and $\left(x^{\prime}, y^{\prime}\right)=\left(x_{0}^{\prime} x_{1}^{\prime} \ldots, y_{0}^{\prime} y_{1}^{\prime} \ldots\right)$ be in $\widetilde{\mathcal{K}} \backslash$ $\operatorname{Max}(\widetilde{\mathcal{K}})$ and such that $\tau(x, y)=\tau\left(x^{\prime}, y^{\prime}\right)$. Let $i$ and $i^{\prime}$ be such that $x_{i} \cdots x_{0} \notin$ $\operatorname{Max}\left(L_{y_{i+1}}\right)$ and $x_{i}^{\prime} \cdots x_{0}^{\prime} \notin \operatorname{Max}\left(L_{y_{i+1}^{\prime}}\right)$. Pose $I=\sup \left\{i, i^{\prime}\right\}$. Thanks to Lemma 5 , $(x, y)$ and $\left(x^{\prime}, y^{\prime}\right)$ do not belong to $\max _{I}$ so the application of $\tau$ will at most affect their prefix of length $I+1$. Since $\tau(x, y)=\tau\left(x^{\prime}, y^{\prime}\right)$, we have $x_{j}=x_{j}^{\prime}$ and $y_{j}=y_{j}^{\prime}$ for all $j>I$. Therefore, $x_{I} \cdots x_{0}$ and $x_{I}^{\prime} \cdots x_{0}^{\prime}$ belongs to $L_{y_{I+1}}=L_{y_{I+1}^{\prime}}$ and have the same successor. So these two words are the same. The conclusion that $y_{I} \cdots y_{0}$ and $y_{I}^{\prime} \cdots y_{0}^{\prime}$ are the same comes from Remark 3 .

Corollary 27. The map $\tau$ is one-to-one from $\widetilde{\mathcal{K}} \backslash \operatorname{Max}(\widetilde{\mathcal{K}})$ onto $\widetilde{\mathcal{K}} \backslash \operatorname{Min}(\widetilde{\mathcal{K}})$.

Proof. It is a direct consequence of the fact that $\tau(\widetilde{\mathcal{K}} \backslash \operatorname{Max}(\widetilde{\mathcal{K}})) \subset \widetilde{\mathcal{K}} \backslash \operatorname{Min}(\widetilde{\mathcal{K}})$. Indeed the restriction of $\tau$ on $\widetilde{\mathcal{K}} \backslash \operatorname{Max}(\widetilde{\mathcal{K}})$ is surjective onto $\widetilde{\mathcal{K}} \backslash \operatorname{Min}(\widetilde{\mathcal{K}})$ since the image of $\operatorname{Max}(\widetilde{\mathcal{K}})$ equals $\{\boldsymbol{0}\} \subset \operatorname{Min}(\widetilde{\mathcal{K}})$.

The topology on $(A \times Q)^{\omega}$ is as usual induced by the distance $d$ defined by

$$
d\left((x, y),\left(x^{\prime}, y^{\prime}\right)\right)=2^{-k} \text { where } k=\inf \left\{i \mid\left(x_{i}, y_{i}\right) \neq\left(x_{i}^{\prime}, y_{i}^{\prime}\right)\right\} .
$$

Proposition 28. The application $\tau$ is continuous on $\widetilde{\mathcal{K}} \backslash \operatorname{Max}(\widetilde{\mathcal{K}})$.

Proof. Let $(u, v) \in \widetilde{\mathcal{K}} \backslash \operatorname{Max}(\widetilde{\mathcal{K}})$ and $\epsilon>0$. We show that there exists $\eta>0$ such that if $d((u, v),(x, y))<\eta$ then $d(\tau(u, v), \tau(x, y))<\epsilon$. Let $j$ be the smallest index such that $(u, v) \notin \max _{j}$. If there exists $i>j$ such that $(u, v)$ and $(x, y)$ have the 
same prefix of length $i+1$ then $\tau(u, v)$ and $\tau(x, y)$ also have the same prefix of length $i+1$. Clearly, one can take

$$
\eta=2^{-\sup \left\{1-\log _{2} \epsilon, j+1\right\}} .
$$

The following example shows that $\tau$ is generally not continuous on the points of $\widetilde{\mathcal{K}} \backslash \operatorname{Max}(\widetilde{\mathcal{K}})$.

Example 29. Consider the regular language $L$ accepted by the automaton depicted in Figure 3 (where the sink is not represented). For instance, $(u, v)=\left(d^{\omega},(12)^{\omega}\right)$

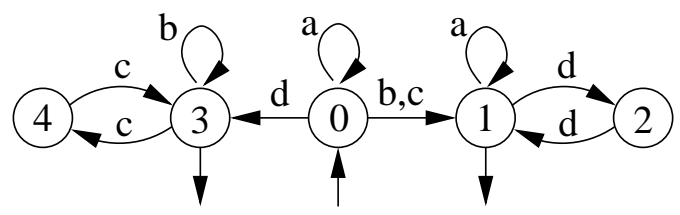

FiguRE 3. The minimal automaton of a language $L$.

belongs to $\operatorname{Max}(\widetilde{\mathcal{K}})$. The points

$$
\left((d d)^{n} b(a)^{\omega},(12)^{n} 1(0)^{\omega}\right) \text { and }\left((d d)^{n} c(a)^{\omega},(12)^{n} 1(0)^{\omega}\right)
$$

can be chosen arbitrarily close of $(u, v)$ for $n$ large enough. Whatever is the value of $\tau(u, v)$, the application $\tau$ is not continuous at $(u, v)$. Indeed,

$$
\tau\left((d d)^{n} b(a)^{\omega},(12)^{n} 1(0)^{\omega}\right)=\left((a a)^{n} c(a)^{\omega},(11)^{n} 1(0)^{\omega}\right)
$$

but

$$
\tau\left((d d)^{n} c(a)^{\omega},(12)^{n} 1(0)^{\omega}\right)=\left((b b)^{n} d(a)^{\omega},(33)^{n} 3(0)^{\omega}\right) .
$$

So clearly, if a point $(x, y)$ is close from an element in $\operatorname{Max}(\widetilde{\mathcal{K}})$ then its image $\tau(x, y)$ is close from an element in $\operatorname{Min}(\widetilde{\mathcal{K}})$ but nothing more can be said.

Remark 30. In the case of positional number systems, such a phenomenon cannot occur: the odometer is always continuous on the points which correspond to $\operatorname{Max}(\widetilde{\mathcal{K}})$.

Proposition 31. The set $\widetilde{\mathcal{K}}$ is a compact subset of $(A \times Q)^{\omega}$. If the odometer $\tau$ is continuous, then the dynamical system $(\widetilde{\mathcal{K}}, \tau)$ is minimal, that is, every non-empty closed subset of $\widetilde{\mathcal{K}}$ invariant under the action of $\tau$ is equal to $\widetilde{\mathcal{K}}$.

Proof. We follow here the proof of [19] adapted to our situation. The compactness of $\widetilde{\mathcal{K}}$ is immediate as a closed subset of $(A \times Q)^{\omega}$.

We assume that $\tau$ is continuous. Let us prove that the closure of the orbit $\left\{\tau^{n}(x, y) \mid n \in \mathbb{N}\right\}$ of any point $(x, y) \in \widetilde{\mathcal{K}}$ is equal to $\widetilde{\mathcal{K}}$.

Let us first observe that the orbit $\left\{\tau^{n}(\mathbf{0}) \mid n \in \mathbb{N}\right\}$ of $\mathbf{0}$ is dense in $\widetilde{\mathcal{K}}$. Indeed, let $(x, y) \in \widetilde{\mathcal{K}}$. Let $\left(w_{n}\right)_{n \in \mathbb{N}}$ be a sequence of words in $L$ such that $x$ is the limit of the sequence $\left(\widetilde{w_{n}}\right)_{n \in \mathbb{N}}$. Let $l_{n}$ denote the $n$-th word in the ordered language $L$. The point $\tau^{n}(\mathbf{0})$ is by definition equal to

$$
\left(\widetilde{l_{n}}\left(a_{0}\right)^{\omega}, \widetilde{p\left(l_{n}\right)}\left(q_{0}\right)^{\omega}\right)
$$

according to notation of Proposition 7. Hence $(x, y)$ is a limit of elements of $\left\{\tau^{n}(\mathbf{0})\right.$ $n \in \mathbb{N}\}$, and $\widetilde{\mathcal{K}}$, which is a closed set, is the closure of $\left\{\tau^{n}(\mathbf{0}) \mid n \in \mathbb{N}\right\}$.

Now if $(x, y) \in \operatorname{Max}(\widetilde{\mathcal{K}})$, then $\tau(x, y)=\mathbf{0}$ and $\mathbf{0}$ belongs to the orbit $(x, y)$, which implies the desired result. 
Let us suppose that $x \notin \operatorname{Max}(\widetilde{\mathcal{K}})$. Let

$$
D: \widetilde{\mathcal{K}} \backslash \operatorname{Max}(\widetilde{\mathcal{K}}) \rightarrow \mathbb{N}: x \mapsto \sup \left\{k \mid(x, y) \in \max _{k}\right\} .
$$

Let us assume that $D$ takes bounded values on the orbit of $(x, y)$. Let $C$ such that $D\left(\tau^{n}(x, y)\right)<C$, for every $n$. In particular, $(x, y)$ does not have the property $\max _{C}$. By definition of the odometer, after a suitable number of iterations of $\tau$, say $n$, then $\tau^{n}(x, y)$ is easily seen to belong to $\max _{C}$. Hence $D$ does not take bounded values on the orbit of $(x, y)$, which implies that there exists an increasing sequence of integers $\left(n_{k}\right)_{k \in \mathbb{N}}$ such that $\tau^{n_{k}}(x, y) \in \max _{k}$. By compactness of $\widetilde{\mathcal{K}}$, one can extract from $\left(n_{k}\right)_{k \in \mathbb{N}}$ an increasing sequence $\left(m_{k}\right)_{k \in \mathbb{N}}$ such that the sequence $\left(\tau^{m_{k}}(x, y)\right)$ converges; its limit belongs to $\operatorname{Max}(\widetilde{\mathcal{K}})$, according to Lemma 5. By continuity of $\tau,\left(\tau^{m_{k}+1}(x, y)\right)$ converges toward $\mathbf{0}$, which implies that the closure of the orbit of $(x, y)$ contains $\mathbf{0}$ and thus equals $\widetilde{\mathcal{K}}$.

\section{Equivalence With positional Systems}

Let $\left(U_{n}\right)_{n \in \mathbb{N}}$ be a strictly increasing sequence of integers such that $U_{0}=1$. Such a sequence is called a positional number system. We assume furthermore that the set $L=0^{*} \operatorname{rep}_{U}(\mathbb{N})$ of all the greedy representations of the integers is a regular language over a finite alphabet $A_{U}$ (from now on $\operatorname{rep}_{U}(n)$ denotes the $U$-representation of $n$ computed by the greedy algorithm with the most significant digit on the left). The finiteness of $A_{U}$ implies that the ratio $U_{n+1} / U_{n}$ is bounded. In particular, for $L=0^{*} \operatorname{rep}_{U}(\mathbb{N})$ (or equivalently for $\operatorname{rep}_{U}(\mathbb{N})$ ) to be regular, it is shown in [36] that the sequence $\left(U_{n}\right)_{n \in \mathbb{N}}$ must satisfy a linear recurrence relation with constant coefficients. In [21], a sufficient condition is given in terms of the polynomials of the recurrence that $\left(U_{n}\right)_{n \in \mathbb{N}}$ satisfies. (The reader can also see the special case treated in [24].) As an example, the set $\operatorname{rep}_{U}(\mathbb{N})$ is regular whenever the sequence $\left(U_{n}\right)_{n \in \mathbb{N}}$ satisfies a linear recurrence relation whose characteristic polynomial is the minimal polynomial of a Pisot number [4].

In this small section, we study the link between the odometer $\tau_{L}$ built over the language $L$ and the odometer $\tau_{U}$ presented in [19]. Notice that we allow leading zeroes in the greedy representations to obtain a language satisfying hypothesis (2).

Remark 32. Notice that, in this particular setting, as a consequence of the greedy algorithm, if $u v$ belongs to $L$ then $v$ belongs also to $L$.

Proposition 33. Let $\left(U_{n}\right)_{n \in \mathbb{N}}$ be a strictly increasing sequence of integers such that $U_{0}=1, \tau_{U}$ be the odometer associated to this sequence, and let us assume that the language $L=0^{*} \operatorname{rep}_{U}(\mathbb{N})$ associated to the numeration system built upon the sequence $\left(U_{n}\right)_{n \in \mathbb{N}}$ is regular. Let $p_{1}: \widetilde{\mathcal{K}} \rightarrow \widetilde{\mathcal{L}}$ be the projection mapping $(x, y)$ onto $x$. Then the following relation holds on $\widetilde{\mathcal{K}}$ :

$$
p_{1} \circ \tau_{L}=\tau_{U} \circ p_{1} .
$$

Proof. Let us first observe that the set on which $\tau_{U}$ is defined and acts, which is the set of right infinite words satisfying the greedy property (1), is exactly $\widetilde{\mathcal{L}}$, following Remark 32 .

Let $(x, y)=\left(x_{0} x_{1} \ldots, y_{0} y_{1} \ldots\right)$ be an element in $\widetilde{\mathcal{K}} \backslash \operatorname{Max}(\widetilde{\mathcal{K}})$. Thus there exists $i$ such that $(x, y) \notin$ max $_{i}$.

Notice that if $u$ belongs to $L_{q}$, since $\mathcal{M}_{L}$ is accessible, then there exists $v$ such that $v u$ belongs to $L$. So thanks to Remark 32, $u$ also belongs to $L$.

Therefore $x_{i} \cdots x_{0}$ belongs to both $L_{y_{i+1}}$ and $L$. Since $x_{i} \cdots x_{0}$ does not belong to $\operatorname{Max}\left(L_{y_{i+1}}\right)$, then it does not belong to $\operatorname{Max}(L)$ which means that $x_{i} U_{i}+\cdots+x_{0} U_{0}$ is strictly less than $U_{i+1}-1$. We set $x_{i}^{\prime} \cdots x_{0}^{\prime}=\operatorname{Succ}_{y_{i+1}}\left(x_{i} \cdots x_{0}\right)$, so with our 
notation

$$
\tau_{L}\left(x_{0} \cdots x_{i} x_{i+1} \ldots, y\right)=\left(x_{0}^{\prime} \cdots x_{i}^{\prime} x_{i+1} \ldots, y^{\prime}\right)
$$

for some $y^{\prime} \in Q^{\omega}$. We have to show that the successor of the word $x_{i} \cdots x_{0}$ in the genealogically ordered language $L$ is $x_{i}^{\prime} \cdots x_{0}^{\prime}$ which means therefore that

$$
x_{i} U_{i}+\cdots+x_{0} U_{0}+1=x_{i}^{\prime} U_{i}+\cdots+x_{0}^{\prime} U_{0}
$$

and thus $\tau_{U}\left(x_{0} \cdots x_{i} x_{i+1} \ldots\right)=\left(x_{0}^{\prime} \cdots x_{i}^{\prime} x_{i+1} \ldots\right)$. To the contrary, assume that there exists $z_{i} \cdots z_{0} \in L$ such that $x_{i} \cdots x_{0}<z_{i} \cdots z_{0}<x_{i}^{\prime} \cdots x_{0}^{\prime}$. Let $v$ be such that $\delta\left(q_{0}, v\right)=y_{i+1}$. The words $v x_{i} \cdots x_{0}$ and $v x_{i}^{\prime} \cdots x_{0}^{\prime}$ are accepted from $q_{0}$ and satisfy therefore the greedy condition (1). Since $z_{i} \cdots z_{0}<x_{i}^{\prime} \cdots x_{0}^{\prime}, v z_{i} \cdots z_{0}$ satisfies the greedy condition and so it belongs to $L$. Since $\mathcal{M}_{L}$ is deterministic, $z_{i} \cdots z_{0}$ is also accepted from $y_{i+1}$. Therefore $x_{i}^{\prime} \cdots x_{0}^{\prime} \neq \operatorname{Succ}_{y_{i+1}}\left(x_{i} \cdots x_{0}\right)$ which is a contradiction.

Consequently if $p_{1}$ is the projection mapping $(x, y)$ onto $x$ then we have shown that on $\widetilde{\mathcal{K}} \backslash \operatorname{Max}(\widetilde{\mathcal{K}})$, the following holds

$$
p_{1} \circ \tau_{L}=\tau_{U} \circ p_{1} \text {. }
$$

Observe that here, $\mathbf{0}$ is $\left(0^{\omega}, q_{0}^{\omega}\right)$ because $\varepsilon$ is the representation of 0 and belongs to $L$. If $(x, y)$ belongs to $\operatorname{Max}(\widetilde{\mathcal{K}})$ then $\tau_{L}(x, y)=\mathbf{0}$ and it is clear that $\sum_{\ell=0}^{i} x_{\ell} U_{\ell}=$ $U_{i+1}-1$ for an infinite number of indices $i$. Therefore from [19], $\tau_{U}(x)=0^{\omega}$ and the relation $(5)$ holds on the whole set $\widetilde{\mathcal{K}}$.

Remark 34. A characterization of the continuity of the odometer for positional number system is given in [16], in terms of the right subsequentiality of the successor function on $0^{*} L$. We will see in Proposition 45 that we can have $\tau_{L}$ continuous whereas $\tau_{U}$ is not continuous.

\section{Substitution Numeration Systems}

7.1. Definition. Let $\Sigma=\left\{a_{1}, \cdots, a_{d}\right\}$ be an alphabet (here, $\Sigma$ does not need to be totally ordered). Let $\sigma: \Sigma \rightarrow \Sigma^{+}$be a substitution, i.e., a morphism of the free monoid $\Sigma^{*}$ such that $\sigma\left(a_{1}\right) \in a_{1} \Sigma^{+}$. To this substitution, we associate a deterministic automaton $\mathcal{M}_{\sigma}=\left(Q, a_{1}, A, \delta, F\right)$ in the classical way. The set of states is $Q=\Sigma \cup\{s\}$ where a sink state $s \notin Q$ is possibly added to $Q$ in order to make $\mathcal{M}_{\sigma}$ complete when $\sigma$ is not a uniform substitution (a substitution is said to be uniform if the images of all the letters have the same length). The alphabet of the automaton is

$$
A=\left\{0, \ldots, \sup _{a \in \Sigma}|\sigma(a)|-1\right\} .
$$

There is an edge of label $i \in A$ between two states $a$ and $b$, that is, $\delta(a, i)=b$ if and only if the $(i+1)$-th letter in $\sigma(a)$ is $b$. The initial state is $a_{1}$ and all the states are final, i.e., $F=\Sigma$.

In the literature $[8,9,10,11,28,30]$ the notion of prefix automaton (respectively prefix-suffix automaton) can also be found. In this latter case, the label $i$ between $a$ and $b$ is replaced by the prefix of length $i$ of $\sigma(a)$ (if $i=0$ then the prefix is $\varepsilon$ ) (respectively, the prefix of length $i$ of $\sigma(a)$ and the suffix of length $|\sigma(a)|-i-1$ of $\sigma(a))$. It is well-known (see for instance [10,11]) that each integer $n \geq 1$ has a unique decomposition of the form

$$
n=\sum_{i=1}^{\ell}\left|\sigma^{i-1}\left(m_{i}\right)\right|
$$

where $m_{\ell} \cdots m_{1}$ is the label of a path read in the prefix automaton from the initial state $a_{1}$ with $m_{\ell} \neq \varepsilon$. 
Let us recall that $d$ denotes the cardinal of the alphabet $\Sigma$. The incidence matrix of the substitution $\sigma$ is defined as the $d \times d$ matrix whose entry of index $(a, b)$ counts the number of occurrences of the letter $a$ in $\sigma(b)$. The incidence matrix of $\sigma$ coincides with the transpose of the adjacency matrix of the automaton $\mathcal{M}_{\sigma}$.

Since the alphabet $A=\left\{0,1, \ldots, \sup _{a \in \Sigma}|\sigma(a)|-1\right\}$ is totally ordered by the usual ordering on $\mathbb{N}$, we can order the words of the language $L \subset A^{*}$ accepted by $\mathcal{M}_{\sigma}$ using the genealogical ordering. This leads to an abstract numeration system $S=(L, A,<)$ built upon $L$.

\subsection{Equivalence between substitution and abstract numeration systems.}

In this section, we give a new interpretation of the numeration systems built upon a substitution according to (6).

Let $\mathcal{M}_{\sigma}^{\prime}$ be the automaton built upon $\mathcal{M}_{\sigma}$ but having an extra state $a_{0}$ which is the initial state of this new automaton. For $k=2, \ldots,\left|\sigma\left(a_{1}\right)\right|$, we add an edge labeled by $k-1$ from $a_{0}$ to the $k$-th letter of $\sigma\left(a_{1}\right)$. Observe that if $L$ is the language accepted by $\mathcal{M}_{\sigma}$ then $L \backslash 0 A^{*}$ is the language accepted by $\mathcal{M}_{\sigma}^{\prime}$. This kind of construction is also classical and was for instance used in [33]. We denote from now on by $L^{\prime}$ the language accepted by $\mathcal{M}_{\sigma}^{\prime}$.

Example 35. Consider the substitution on $\Sigma=\left\{a_{1}, a_{2}\right\}$ defined by $\sigma\left(a_{1}\right)=a_{1} a_{2} a_{1}$ and $\sigma\left(a_{2}\right)=a_{1}$. We have the following automata $\mathcal{M}_{\sigma}$ and $\mathcal{M}_{\sigma}^{\prime}$ sketched in Figure 4. Here $A=\{0,1,2\}$ and the sink has not been represented.

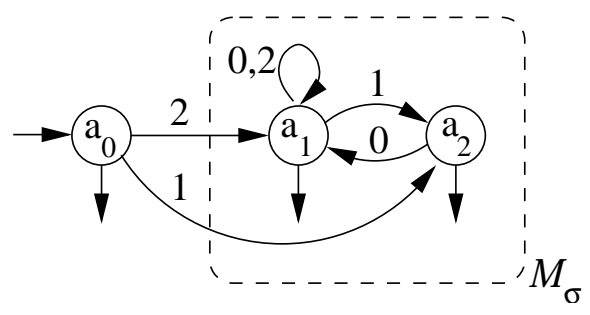

Figure 4 . The automata $\mathcal{M}_{\sigma}$ and $\mathcal{M}_{\sigma}^{\prime}$.

Naturally, we can also order the words of the language $L^{\prime} \subset A^{*}$ accepted by $\mathcal{M}_{\sigma}^{\prime}$ using the genealogical ordering. This leads to an abstract numeration system $S^{\prime}=\left(L^{\prime}, A,<\right)$ built upon $L^{\prime}$. The representation of the integer $n$ is defined as the $(n+1)$-th word $w$ of $L^{\prime}$ and we write $\operatorname{val}_{S^{\prime}}(w)=n$ (let us recall that the first word of $L^{\prime}$ is the empty word).

The following proposition allows us to make the connection with the substitutive numeration system as expressed in (6).

Proposition 36. The $(n+1)$ th word $w_{1} \cdots w_{\ell}$ of the genealogically ordered language $L^{\prime}$ generates the prefix $u_{0} \cdots u_{n-1}$ of length $n$ of $\sigma^{\omega}\left(a_{1}\right)$ as follows: $u_{0} \cdots u_{n-1}$ is equal to the concatenation of $\sigma^{\ell-i}\left[\delta\left(a_{1}, w_{1} \cdots w_{i-1} 0\right) \cdots \delta\left(a_{1}, w_{1} \cdots w_{i-1}\left(w_{i}-1\right)\right)\right]$ in decreasing order of indices $1 \leq i \leq \ell$, where $w_{1} \cdots w_{i-1}$ is understood as $\varepsilon$ if $i=1$, as well as $\delta\left(a_{1}, w_{1} \cdots w_{i} 0\right) \cdots \bar{\delta}\left(a_{1}, w_{1} \cdots w_{i}\left(w_{i}-1\right)\right)$ if $w_{i}=0$. In other words, $u_{0} \cdots u_{n-1}$ is equal to

$\sigma^{\ell-1}\left[\delta\left(a_{1}, 0\right) \cdots \delta\left(a_{1},\left(w_{1}-1\right)\right)\right] \cdots \sigma^{0}\left[\delta\left(a_{1}, w_{1} \cdots w_{\ell-1} 0\right) \cdots \delta\left(a_{1}, w_{1} \cdots w_{l-1}\left(w_{\ell}-1\right)\right)\right]$

and

$$
n=\sum_{i=0}^{\ell-1}\left|\sigma^{i}\left[\delta\left(a_{1}, w_{1} \cdots w_{i-1} 0\right) \cdots \delta\left(a_{1}, w_{1} \cdots w_{i-1}\left(w_{i}-1\right)\right)\right]\right|
$$


Proof. The proof is based on the fact that the prefix of $\sigma(q)$ of length $t \leq|\sigma(q)|$ read from the state $q \in \Sigma$ is equal to $\delta(q, 0) \cdots \delta(q, t-1)$.

Let us recall that for a state $q, \mathbf{u}_{q}(n)$ denotes the cardinal of the set of the words of length $n$ accepted from $q$. If $w=w_{1} \cdots w_{\ell} \in L^{\prime}$ (this means in particular that $w_{1}>0$ ), then with respect to the automaton $\mathcal{M}_{\sigma}^{\prime}$ the following formula holds (see $[22,23])$

$$
\begin{aligned}
\operatorname{val}_{S^{\prime}}(w)= & \sum_{i=0}^{\ell-1} \mathbf{u}_{a_{0}}(i)+\sum_{b<w_{1}} \mathbf{u}_{\delta\left(a_{0}, b\right)}(\ell-1) \\
& +\sum_{b<w_{2}} \mathbf{u}_{\delta\left(a_{0}, w_{1} b\right)}(\ell-2)+\cdots+\sum_{b<w_{\ell}} \mathbf{u}_{\delta\left(a_{0}, w_{1} \cdots w_{\ell-1} b\right)}(0) .
\end{aligned}
$$

The interested reader can find a combinatorial interpretation of this formula in [20]. We have two immediate observations

$$
\sum_{i=0}^{\ell-1} \mathbf{u}_{a_{0}}(i)=\mathbf{u}_{a_{1}}(\ell-1) \quad \text { and } \quad \forall q \in \Sigma, \forall n \in \mathbb{N}, \mathbf{u}_{q}(n)=\left|\sigma^{n}(q)\right| .
$$

We are now able to prove the equivalence of the two formulas (6) and (7). First notice that $\delta\left(a_{0}, 0\right)$ is the sink $s$ of $\mathcal{M}_{\sigma}^{\prime}$. Therefore $\mathbf{u}_{\delta\left(a_{0}, 0\right)}(n)=0$ for all $n$. If $b \neq 0$ then $\delta\left(a_{0}, b\right)=\delta\left(a_{1}, b\right)$. The first two terms in (7) can be written as

$$
\left|\sigma^{\ell-1}\left(a_{1}\right)\right|+\sum_{0<b<w_{1}}\left|\sigma^{\ell-1}\left[\delta\left(a_{1}, b\right)\right]\right|=\left|\sigma^{\ell-1}\left[\delta\left(a_{1}, 0\right) \cdots \delta\left(a_{1}, w_{1}-1\right)\right]\right| .
$$

Notice that for the latter equality, we have used the fact that $\delta\left(a_{1}, 0\right)=a_{1}$ and that $\sigma$ is a morphism. Consequently, (7) can be written as

$$
\operatorname{val}_{S^{\prime}}(w)=\sum_{i=1}^{\ell}\left|\sigma^{\ell-i}\left[\delta\left(a_{1}, w_{1} \cdots w_{i-1} 0\right) \cdots \delta\left(a_{1}, w_{1} \cdots w_{i-1}\left(w_{i}-1\right)\right)\right]\right| .
$$

This gives another interpretation of (6).

7.3. First properties of the odometer. Since the infinite language $L$ accepted by $\mathcal{M}_{\sigma}$ satisfies property (2), then one can consider the odometer $\widetilde{\mathcal{K}}$ built upon $(L, A,<)$. Let us observe that $L^{\prime}$ does not satisfy $(2)$, but that that $\widetilde{\mathcal{L}}$ and $\widetilde{\mathcal{L}^{\prime}}$ do coincide. The set $\widetilde{\mathcal{K}}$ is a subshift of finite type of $(A \times Q)^{\omega}$ since every state (except the sink) in $\mathcal{M}_{\sigma}$ is a final state. Let us observe

Proposition 37. The set $\widetilde{\mathcal{K}}$ corresponds to the set of infinite paths $(x, y) \in(A \times$ $\Sigma)^{\omega}$ in the automaton $\widetilde{\mathcal{M}}_{\sigma}$, i.e., $\forall i \geq 0, y_{i+1}$ is the $\left(x_{i}+1\right)$-th letter of $\sigma\left(y_{i}\right)$. Furthermore,

$$
\begin{gathered}
\operatorname{Max}(\widetilde{\mathcal{K}})=\left\{(x, y) \in \widetilde{\mathcal{K}}\left|\forall i \geq 0, x_{i}=\right| \sigma\left(y_{i}\right) \mid-1\right\}, \\
\operatorname{Min}(\widetilde{\mathcal{K}})=\left\{(x, y) \in \widetilde{\mathcal{K}} \mid \forall i \geq 0, x_{i}=0\right\} .
\end{gathered}
$$

Proof. There is an edge in the automaton $\widetilde{\mathcal{M}}_{\sigma}$ of label $i$ between two states $u$ and $v$ if and only if the $(i+1)$-th letter in $\sigma(v)$ is $u$; furthermore, all the states are initial and final. Hence $\widetilde{\mathcal{L}}$ is equal to the set of labels of infinite paths in $\widetilde{\mathcal{M}}_{\sigma}$, which implies the desired description of $\widetilde{\mathcal{K}}$. The characterization of $\operatorname{Max}(\widetilde{\mathcal{K}})$ and $\operatorname{Min}(\widetilde{\mathcal{K}})$ is immediate.

Let us recall that the odometer $\tau$ is one-to-one from $\widetilde{\mathcal{K}} \backslash \operatorname{Max}(\widetilde{\mathcal{K}})$ onto $\widetilde{\mathcal{K}} \backslash \operatorname{Min}(\widetilde{\mathcal{K}})$ following Corollary 27. 
Remark 38. The automaton $\mathcal{M}_{\sigma}$ associated to a substitution $\sigma$ is not necessarily minimal. Indeed, if you consider the Thue-Morse substitution defined by $\sigma\left(a_{1}\right)=$ $a_{1} a_{2}$ and $\sigma\left(a_{2}\right)=a_{2} a_{1}$ then it is easy to see that $\mathcal{M}_{\sigma}$ accept $\{0,1\}^{*}$ and is not minimal. To obtain unambiguous constructions, we have always considered the minimal automaton of a language but clearly, we could define a set $\widetilde{\mathcal{K}}$ and an odometer $\tau$ depending on the choice of a finite deterministic automaton which is not necessarily minimal. We just need a loop in the initial state $a_{1}$ labeled by the smallest letter 0 of the alphabet (it is always the case for the automaton associated to a substitution $\sigma$ which satisfies $\sigma\left(a_{1}\right) \in a_{1} A^{+}$).

We can say nothing in general concerning the continuity of $\tau$ as illustrated in Proposition 45.

7.4. The Pisot case. Nevertheless, there are some cases for which the automaton $\mathcal{M}_{\sigma}$ can be proved to be minimal. A substitution is said of Pisot type if the eigenvalues of its incidence matrix satisfy the following: there exists a dominant eigenvalue $\alpha$ such that for every other eigenvalue $\lambda$, one gets $0<|\lambda|<1<|\alpha|$. A substitution of Pisot type is primitive and the characteristic polynomial $\chi_{\sigma}$ of its incidence matrix is irreducible over $\mathbb{Q}[9]$. Let us recall that $\chi_{\sigma}$ is also the minimal polynomial of the adjacency matrix of $\mathcal{M}_{\sigma}$.

Proposition 39. Let $\sigma$ be a Pisot substitution. The automaton $\mathcal{M}_{\sigma}$ is minimal.

Proof. Let $\sigma$ be a substitution of Pisot type. The automaton $\mathcal{M}_{\sigma}$ is accessible since $\sigma$ is primitive, that is, all its states can be reached from its initial state $a_{1}$. Hence the minimal polynomial $\chi_{\sigma}$ of its adjacency matrix is dividable by the minimal polynomial of the minimal automaton recognizing the language $M_{\sigma}$. Since $\chi_{\sigma}$ is irreducible, this implies that both polynomials do coincide, and thus that $\mathcal{M}_{\sigma}$, which is deterministic, is the minimal automaton recognizing the language $M_{\sigma}$.

In the particular case of a Pisot substitution, we are now able to give a dynamical interpretation of $(\widetilde{\mathcal{K}}, \tau)$. Let $S$ denote the shift map on $\Sigma^{\mathbb{Z}}: S\left(\left(w_{i}\right)_{i \in \mathbb{Z}}\right)=\left(w_{i+1}\right)_{i \in \mathbb{Z}}$. A word $u \in \Sigma^{\mathbb{Z}}$ such that there exists a positive integer $k$ with $S^{k}(u)=u$ is called a periodic point under the action of $\sigma$. Let us recall that the (two-sided) symbolic dynamical system generated by a primitive substitution $\sigma$ is the pair $\left(X_{\sigma}, S\right)$, where $X_{\sigma}$ is the set of two-sided sequences in $\Sigma^{\mathbb{Z}}$ with the same set of factors of any periodic point $u$ of $\sigma$; this definition does not depend on the choice of $u$ by primitivity of $\sigma$.

We use here the notation and results of $[8,9]$ adapted to our framework. Following [26], every two-sided sequence $v$ in $X_{\sigma}$ has a unique decomposition

$$
v=S^{k}(\sigma(w)), \text { with } w \in X_{\sigma} \text { and } 0 \leq k<\left|\sigma\left(w_{0}\right)\right|
$$

( $w_{0}$ denotes here the 0 -th coordinate of $w$ ).

Let

$$
\left\{\begin{array}{l}
\theta: X_{\sigma} \rightarrow X_{\sigma}: v \mapsto w, \\
\text { where } v=S^{k}(\sigma(w)), \text { with } 0 \leq k<\left|\sigma\left(w_{0}\right)\right| .
\end{array}\right.
$$

The map $\theta$ is called the desubstitution map.

Let

$$
\left\{\begin{array}{l}
\gamma: X_{\sigma} \rightarrow(A \times \Sigma): v \mapsto\left(k+1, w_{0}\right) \\
\text { where } v=S^{k}(\sigma(w)), \text { with } w \in X_{\sigma} \text { and } 0 \leq k<\left|\sigma\left(w_{0}\right)\right| .
\end{array}\right.
$$

In other words, if $\gamma(v)=(k, q)$, then $v_{0}$ is the $(k+1)$-th letter of $\sigma(q)$. Hence, for every $v \in X_{\sigma}$, the sequence $\left(\gamma \circ \theta^{i}(v)\right)_{i \in \mathbb{N}}$ is easily seen to belong to $\widetilde{\mathcal{K}}$. Let us now define

$$
\Gamma: X_{\sigma} \rightarrow \widetilde{\mathcal{K}}: v \mapsto\left(\gamma \circ \theta^{i}(v)\right)_{i \in \mathbb{N}} .
$$

The following theorem is a direct consequence of $[8,9]$. 
Theorem 40. $[8,9]$ Let $\sigma$ be a Pisot substitution. The map $\Gamma$ is continuous and onto $\widetilde{\mathcal{K}}$; it is one-to-one except on the orbit of periodic points of $\sigma$. Furthermore,

$$
\Gamma \circ S=\tau \circ \Gamma \text { and } \Gamma \circ \theta=S_{\widetilde{\mathcal{K}}^{\circ}} \Gamma,
$$

where $S_{\widetilde{\mathcal{K}}}$ denotes the shift map acting on elements of $(A \times \Sigma)^{\mathbb{Z}}$.

Proof. We know from [8] that $\left(X_{\sigma}, S\right)$ is measure-theoretically isomorphic with the subshift of finite type $\mathcal{D}$ defined as the set of labels of infinite paths $\mathcal{D}$ in the prefix-suffix automaton. Let us recall that there is an edge from $a$ to $b$ of label $(p, a, s)$ in the prefix-suffix automaton if $\sigma(b)=a$, and all its states (which are the letters of $\Sigma$ ) are both initial and final. Let us prove that $\widetilde{\mathcal{K}}$ and $\mathcal{D}$ are in one-to-one correspondence. This comes from the fact that the following map is one-to-one:

$$
\widetilde{\mathcal{K}} \rightarrow \mathcal{D},(x, y) \mapsto\left(\delta\left(y_{i+1}, 0\right) \cdots \delta\left(y_{i+1}, x_{i}-1\right), y_{i}, s_{i}\right)_{i \in \mathbb{N}},
$$

where $s_{i}$ is the suffix of size $\left|\sigma\left(y_{i+1}\right)\right|-x_{i}-1$ of $\sigma\left(y_{i+1}\right)$. Now from Remark 23, the map $\tau$ coincides with the adic transformation acting on $\mathcal{D}$. It just remains to apply the results of $[8,9]$.

Remark 41. Two dynamical systems can be built over $\widetilde{\mathcal{K}}$, i.e., $(\widetilde{\mathcal{K}}, \tau)$ and $\left(\widetilde{\mathcal{K}}, S_{\widetilde{\mathcal{K}}}\right)$. Theorem 40 gives us two combinatorial interpretations for these systems: the action of the desubstitution map $\theta$ (the "inverse" of $\sigma$ ) on $X_{\sigma}$ corresponds to the action of the shift $S_{\widetilde{\mathcal{K}}}$ on $\widetilde{\mathcal{K}}$, whereas the action of the shift $S$ on $X_{\sigma}$ corresponds to action of the odometer $\tau$ on $\widetilde{\mathcal{K}}$.

Remark 42. Furthermore, there exists a unique shift invariant measure on the dynamical system $\left(X_{\sigma}, S\right)$ since $\sigma$ is primitive $\left(\left(X_{\sigma}, S\right)\right.$ is said uniquely ergodic); for more details see for instance [29]. This measure can be naturally carried on $(\widetilde{\mathcal{K}}, \tau)$ via the map $\Gamma$ (which is one-to-one except on a countable number of points). Theorem 40 means that $(\widetilde{\mathcal{K}}, \tau)$ endowed with this measure is measure-theoretically isomorphic with $\left(X_{\sigma}, S\right)$. One interest of this approach is that it provides us some insight on a metrical study of $(\widetilde{\mathcal{K}}, \tau)$, following [6].

Remark 43. It is possible to give a combinatorial interpretation of $\operatorname{Min}(\widetilde{\mathcal{K}})$ and $\operatorname{Max}(\widetilde{\mathcal{K}})$ in this framework. Following [8], $\operatorname{Min}(\widetilde{\mathcal{K}})$ and $\operatorname{Max}(\widetilde{\mathcal{K}})$ correspond respectively to the periodic points (under the action of $\sigma$ ) of $X_{\sigma}$ (we denote this set $\left.\operatorname{Per}\left(X_{\sigma}\right)\right)$ and to the preimages $S^{-1}\left(\operatorname{Per}\left(X_{\sigma}\right)\right)$ under the shift $S$ of those periodic points. Both sets do not have necessarily the same cardinal as illustrated for instance in Section 8, Proposition 45.

\section{THE CASE OF SOFiC BETA-NUMERATIONS}

This section gathers results of Section 6 and 7 within the framework of $\beta$ numeration. Let $U=\left(U_{n}\right)_{n \in \mathbb{N}}$ be a positional numeration system such that the ratio $U_{n+1} / U_{n}$ is bounded, as defined in Section 6. Assume now that $\operatorname{rep}_{U}(\mathbb{N}) 0^{*}$ is included in $\operatorname{rep}_{U}(\mathbb{N})$; the positional number system $U$ is said to be a Bertrand numeration system. Bertrand numeration systems are closely related to $\beta$-expansions as recalled below.

Let $\beta>1$ be a positive real number. The Rényi $\beta$-expansion of a real number $x \in$ $[0,1]$ is defined as the sequence $\left(x_{i}\right)_{i \geq 1}$ with values in $\{0,1, \ldots,\lceil\beta\rceil-1\}$ produced by the $\beta$-transformation $T_{\beta}:[0,1] \rightarrow[0,1]: x \mapsto \beta x(\bmod 1)$ as follows

$$
\forall i \geq 1, x_{i}=\left\lfloor\beta T_{\beta}^{i-1}(x)\right\rfloor \text {, and thus } x=\sum_{i \geq 1} x_{i} \beta^{-i} \text {. }
$$


Let $d_{\beta}(1)=\left(t_{i}\right)_{i>1}$ denote the $\beta$-expansion of 1 . Let $d_{\beta}^{*}(1)=d_{\beta}(1)$, if $d_{\beta}(1)$ is infinite, and $d_{\beta}^{*}(1)=\left(t_{1} \ldots t_{m-1}\left(t_{m}-1\right)\right)^{\omega}$, if $d_{\beta}(1)=\left(t_{1} \ldots t_{m-1} t_{m}\right)$ is finite (with $\left.t_{m} \neq 0\right)$. The set $D_{\beta}$ of $\beta$-expansions of numbers in $[0,1)$ is exactly the set of sequences $\left(c_{i}\right)_{i \geq 1}$ that satisfy:

$$
\forall k \in \mathbb{Z},\left(c_{i}\right)_{i \geq k}<_{\operatorname{lex}} d_{\beta}^{*}(1) .
$$

For more details, see for instance [25]. We denote by $F\left(D_{\beta}\right)$ the set of finite factors of the sequences in $D_{\beta}$.

Numbers $\beta$ such that $d_{\beta}(1)$ is ultimately periodic are called $\beta$-numbers and those such that $d_{\beta}(1)$ is finite are called simple $\beta$-numbers. If $\beta$ is a $\beta$-number (simple or not), the minimal automaton $\mathcal{M}_{\beta}$ recognizing the set of factors of $F\left(D_{\beta}\right)$ can easily be constructed (representations of this classical automaton $\mathcal{M}_{\beta}$ can be found in [18] or [23]). Furthermore, let us recall that when $\beta$ is assumed to be Pisot, then $\beta$ is either a $\beta$-number or a simple $\beta$-number, and $\left(X_{\beta}, S\right)$ is sofic.

Bertrand numeration systems are characterized by the following theorem:

Theorem 44. [3] Let $U$ be a positional number system over a finite alphabet. Then $U$ is a Bertrand numeration system if and only if there exists a real number $\beta>1$ such that $L=0^{*} \operatorname{rep}_{U}(\mathbb{N})=F\left(D_{\beta}\right)$. Furthermore, $L$ is regular if and only if $\beta$ is a $\beta$-number.

There is a natural way to associate a substitution $\sigma_{\beta}$ with the $\beta$-numeration when $\beta$ is a $\beta$-number (simple or not). These substitutions will be called in all what follows $\beta$-substitutions. The automaton $\mathcal{M}_{\sigma_{\beta}}$ associated with $\sigma_{\beta}$, as defined in Section 7 , coincides with the minimal automaton $\mathcal{M}_{\beta}$ which recognizes $F\left(D_{\beta}\right)$. For more details, see $[13,39]$. Let us note that $d_{\beta}(1)$ cannot be purely periodic, hence one has either $d_{\beta}^{*}(1)=\left(t_{1} \cdots t_{n-1}\left(t_{n}-1\right)\right)^{\omega}$ with $t_{n} \neq 0$ or $d_{\beta}^{*}(1)=t_{1} \cdots t_{n}\left(t_{n+1} \cdots t_{n+p}\right)^{\omega}$, with $t_{n} \neq t_{n+p}$ and $n \geq 1$.

- Assume $d_{\beta}(1)=\left(t_{1} \cdots t_{n-1} t_{n}\right)$ with $t_{n} \neq 0$ and thus $d_{\beta}^{*}(1)=\left(t_{1} \cdots t_{n-1}\left(t_{n}-\right.\right.$ 1) $)^{\omega}$. Consider the substitution $\sigma_{\beta}$ defined over the alphabet $\{1,2, \ldots, n\}$ by:

$$
\sigma_{\beta}: \begin{cases}1 & \mapsto 1^{t_{1}} 2 \\ 2 & \mapsto 1^{t_{2}} 3 \\ \vdots & \vdots \\ n-1 & \mapsto 1^{t_{n}-1} n \\ n & \mapsto 1^{t_{n}} .\end{cases}
$$

- Assume $d_{\beta}(1)=d_{\beta}^{*}(1)=t_{1} \cdots t_{n}\left(t_{n+1} \cdots t_{n+p}\right)^{\omega}$, with $t_{n+1} \cdots t_{n+p} \neq 0^{p}$ and $t_{n} \neq t_{n+p}$. Furthermore $n \geq 1$. Consider the substitution $\sigma_{\beta}$ defined over the alphabet $\{1,2, \ldots, n+p\}$ by:

$$
\sigma_{\beta}: \begin{cases}1 & \mapsto 1^{t_{1}} 2 \\ 2 & \mapsto 1^{t_{2}} 3 \\ \vdots & \vdots \\ n+p-1 & \mapsto 1^{t_{n+p-1}}(n+p) \\ n+p & \mapsto 1^{t_{n+p}}(n+1) .\end{cases}
$$

¿From now on, we assume that the positional number system $U$ is a Bertrand numeration associated with $\beta$ Pisot number; thus $L=0^{*} \operatorname{rep}_{U}(\mathbb{N})=F\left(D_{\beta}\right)$ is an infinite regular language which satisfies $(2)$. We still denote $\tau_{L}$ the odometer acting on $\widetilde{\mathcal{K}}$. When $\beta$ is a simple $\beta$-number, then the substitutions $\sigma_{\beta}$ are of Pisot type since the characteristic polynomial of their incidence matrices coincides with the minimal polynomial of $\beta$. Hence, the results of Section 7.4 do apply. 
We end now this section by proving that the odometer $\tau_{L}$ is continuous when $\beta$ is a Pisot number, contrarily to the positional number systems case where continuity holds if and only if $\beta$ is a simple $\beta$-number (see [19] and [16]).

Proposition 45. Let $\beta$ be a Pisot number and let $L=F\left(D_{\beta}\right)$. Then the odometer $\tau_{L}$ is continuous on $\widetilde{\mathcal{K}}$.

Proof. Let $(x, y) \in \operatorname{Max}(\widetilde{\mathcal{K}})$. Let us prove that for any sequence $\left(x^{(n)}, y^{(n)}\right)_{n \in \mathbb{N}}$ with values in $\widetilde{\mathcal{K}}$ which converges toward $(x, y)$, then $\tau_{L}\left(x^{(n)}, y^{(n)}\right)_{n \in \mathbb{N}}$ converges toward $\mathbf{0}=\left(0^{\omega}, q_{0}^{\omega}\right)$. Let $\left(x^{(n)}, y^{(n)}\right)_{n \in \mathbb{N}}$ be such a sequence. We assume furthermore that for $n$ large enough, then $\left(x^{(n)}, y^{(n)}\right) \notin \operatorname{Max}(\widetilde{\mathcal{K}})$. There exists a state $q$ for which there exist infinitely many integers $k$ such that $x_{k} x_{k-1} \cdots x_{0} \in \operatorname{Max}\left(L_{q}\right)$. Let $N$ be fixed. Let $k \geq N$ such that $x_{k} x_{k-1} \cdots x_{0} \in \operatorname{Max}\left(L_{q}\right)$ with $q=y_{k+1}$. For $n$ large enough, $\left(x^{(n)}, y^{(n)}\right)$ coincides on its first $N$ values with $(x, y)$ and $\left(x^{(n)}, y^{(n)}\right) \notin \operatorname{Max}(\widetilde{\mathcal{K}})$. In particular, $x_{k}^{(n)} x_{k-1}^{(n)} \cdots x_{0}^{(n)} \in \operatorname{Max}\left(L_{q}\right)$, with $q=y_{k+1}^{(n)}$. Since $\left(x^{(n)}, y^{(n)}\right) \notin \operatorname{Max}(\widetilde{\mathcal{K}})$, there exists a non-negative integer $l>k$ such that $x_{l}^{(n)} x_{l-1}^{(n)} \cdots x_{0}^{(n)} \notin \operatorname{Max}\left(L_{y_{l+1}^{(n)}}\right)$. Let $l_{0}$ denote the smallest of these integers. The successor in $L_{y_{l_{0}+1}^{(n)}}$ of $x_{l_{0}}^{(n)} x_{l_{0}-1}^{(n)} \cdots x_{0}^{(n)}$ is $\left(x_{l_{0}}^{(n)}+1\right) 0^{l_{0}}$. Furthermore, any edge labeled by 0 in $\mathcal{M}_{\beta}$ leads to the initial state $q_{0}$ (recall that the interested reader can find a representation of $\mathcal{M}_{\beta}$ in $\left.[18,23]\right)$. Hence $\tau_{L}\left(x^{(n)}, y^{(n)}\right)$ admits as a prefix $\left(0^{N}, y_{0}^{N}\right)$ for $n$ large enough, which ends the proof.

Remark 46. The sets $\widetilde{\mathcal{K}}$ and $\widetilde{\mathcal{L}}$ are not in one-to-one correspondence. Indeed the word $0^{\omega}$ admits several representations in $\widetilde{\mathcal{K}}$, hence we cannot deduce directly continuity results from Proposition 33. Let us observe nevertheless that there is at most a countable number of antecedents to elements of $\widetilde{\mathcal{L}}$ according to the projection $p_{1}: \widetilde{\mathcal{K}} \rightarrow \widetilde{\mathcal{L}},(x, y) \mapsto x$, in the particular situation described in this section.

\section{Real Representation of the odometer}

The aim of this section is to outline the first steps of a study of a geometric representation of the dynamical system $(\widetilde{\mathcal{K}}, \tau)$. A geometric representation of the dynamical system $(\widetilde{\mathcal{K}}, \tau)$ is a continuous map $\varphi$ from $\widetilde{\mathcal{K}}$ onto a geometric dynamical system $(Y, T)$ such that $\varphi \circ \tau=T \circ \varphi$, and on which there exists a partition indexed by the alphabet $A \times Q$ such that every word $(x, y)$ is the itinerary of a point of $(Y, T)$ with respect to the partition.

Let $L$ be an arbitrary regular language satisfying (2). A first representation which might be possible consists in extending the work of [22, 23, 34], where a real value is attributed to limits of finite words for abstract numeration systems built on an exponential regular language satisfying the following conditions: there exist $\beta>1$ and $P \in \mathbb{R}[X]$ such that for all states $q \in Q$, there exists some nonnegative real numbers $a_{q}$ such that $\lim _{n \rightarrow \infty} \frac{\mathbf{u}_{q}(n)}{P(n) \beta^{n}}=a_{q}$. (We recall that $\mathbf{u}_{q}(n)$ represents the number of words of length $n$ in $L_{q}$.) We assume now that $\widetilde{L}$ also satisfies the previous conditions of $[22,23]$. Clearly, if $L$ is exponential then $\widetilde{L}$ is also exponential because $\#\left(L \cap \Sigma^{n}\right)=\#\left(\widetilde{L} \cap \Sigma^{n}\right)$. The main assumptions for building a representation map rely therefore on the asymptotic behavior of the sequences $\frac{\mathbf{u}_{q^{\prime}}(n)}{P(n) \beta^{n}}$ for all the states $q^{\prime}$ of the minimal automaton of $\widetilde{L}$. Let $\mathbf{v}(n)$ denotes the number of words of length at most $n$ in $L$ (or in $\widetilde{L}$ ), and $\operatorname{val}_{\widetilde{L}}(w)$ the numerical value of $w \in \widetilde{L}$, i.e., if $\operatorname{val}_{\tilde{L}}(w)=n$, then $w$ is the $(n+1)$-th word of $\widetilde{L}$. Let $(x, y) \in \widetilde{\mathcal{K}}$. Since $x \in \widetilde{\mathcal{L}}$, there exists a sequence $\left(w_{n}\right)_{n \in \mathbb{N}}$ of words in $\widetilde{L}$ which converges to $x$. 
The limit

$$
\lim _{n \rightarrow \infty} \frac{\operatorname{val}_{\widetilde{L}}\left(w_{n}\right)}{\mathbf{v}\left(\left|w_{n}\right|\right)}
$$

does not depend on the choice of the sequence $\left(w_{n}\right)_{n \in \mathbb{N}}$ (see [22, Corollary 8]) and is denoted by $\operatorname{val}_{\infty}(x)$. We thus can define a map $r: \widetilde{\mathcal{K}} \rightarrow \mathbb{R}:(x, y) \mapsto \operatorname{val}_{\infty}(x)$. It remains to explore the properties of such a representation map $r$.

We propose now a second possible geometric representation in the framework of languages associated with substitutions. We continue here with the notation of Section 7. A substitution is said unimodular if the determinant of its incidence matrix equals \pm 1 . It is conjectured that for a Pisot unimodular substitution, the dynamical system $\left(X_{\sigma}, S\right)$, and hence $(\widetilde{\mathcal{K}}, \tau)$ according to Remark 42 , is measure-theoretically isomorphic to a rotation on the torus $\mathbb{T}^{d-1}$, where $d$ denotes the cardinal of the alphabet $\Sigma$. For more details, see for instance Chap. 7 of [28]. There are however families of substitutions for which this result is known to hold true. One simple way to exhibit this rotation is to give a geometrical representation of $(\widetilde{\mathcal{K}}, \tau)$ as explained in the next paragraph. We follow the formalism of $[8,9]$.

Let $L$ be a language associated with the substitution $\sigma$ of Pisot type over the alphabet $\Sigma$ of cardinal $d$, as explained in Section 7 . Let us recall that the characteristic polynomial $\chi_{\sigma}$ of the incidence matrix of $\sigma$ is also the minimal polynomial of the adjacency matrix of the minimal automaton $\mathcal{M}_{L}$ recognizing $L$. Let $\alpha_{1}, \ldots, \alpha_{r}$ denote the $r$ real eigenvalues of $\chi_{\sigma}$, and $\alpha_{r+1}, \ldots, \alpha_{r+s}, \overline{\alpha_{r+1}}, \ldots, \overline{\alpha_{r+s}}$, denote its $2 s$ complex roots $(r+2 s=d)$. Let us assume $\alpha_{1}>1$, hence, $\left|\alpha_{i}\right|<1$, for $i \geq 2$.

Let $\vec{v}^{(1)}$ be a left eigenvector associated with the eigenvalue $\alpha_{1}$ with coefficients in the field $\mathbb{Q}\left(\alpha_{1}\right)$. Let $\alpha_{k}$ be an eigenvalue and let $\rho_{k}$ be the canonical morphism from $\mathbb{Q}\left(\alpha_{1}\right)$ onto $\mathbb{Q}\left(\alpha_{k}\right)$, extended to $\mathbb{Q}\left(\alpha_{1}\right)^{d}$. Let $\vec{v}^{(k)}=\rho_{k}\left(\vec{v}^{(1)}\right)$. We propose as a geometric representation of the set $\widetilde{\mathcal{K}}$ in this framework the following map $\varphi: \widetilde{\mathcal{K}} \rightarrow \mathbb{R}^{r-1} \times \mathbb{C}^{s}:$

$(x, y) \mapsto\left(\sum_{i \geq 0}\left(\vec{v}_{\delta\left(y_{i+1}, 0\right)}^{(2)}+\cdots+\vec{v}_{\delta\left(y_{i+1}, x_{i}-1\right)}^{(2)}\right) \alpha_{2}^{i}, \cdots,\left(\vec{v}_{\delta\left(y_{i+1}, 0\right)}^{(r+s)}+\cdots+\vec{v}_{\delta\left(y_{i+1}, x_{i}-1\right)}^{(r+s)}\right) \alpha_{r+s}^{i}\right)$.

This series is easily seen to converge. This map can be factorized as a map on the torus. Indeed, let $\mathbb{L}$ denote the lattice

$$
\left\{\sum_{k=1}^{d} n_{k} \vec{v}^{(k)} \mid n_{k} \in \mathbb{Z}, \sum_{k=1}^{d} n_{k}=0\right\}
$$

Following $[8,9]$, the map

$$
\varphi_{\mathbb{L}}: \widetilde{\mathcal{K}} \rightarrow\left(\mathbb{R}^{r-1} \times \mathbb{C}^{s}\right) / \mathbb{L} \equiv \mathbb{T}^{d-1},(x, y) \mapsto \varphi(x, y) \bmod \mathbb{L}
$$

is well defined and continuous. Consider the toral translation

$$
T:\left(\mathbb{R}^{r-1} \times \mathbb{C}^{s}\right) / \mathbb{L} \rightarrow\left(\mathbb{R}^{r-1} \times \mathbb{C}^{s}\right) / \mathbb{L}: z \mapsto z+\vec{v}^{(1)} \bmod \mathbb{L} .
$$

One checks that $\varphi_{\mathbb{L}} \circ \tau=T \circ \varphi_{\mathbb{L}}$, and that $\varphi$ is a geometrical representation of $(\widetilde{\mathcal{K}}, \tau)$, the partition being given by the sets $\varphi\left\{(x, y) \mid(x, y) \in \widetilde{\mathcal{K}}, y_{0}=q\right\}, q \in Q$.

In particular, for some families of $\beta$-substitutions, this map is known to provide a measure-theoretical isomorphism (this is the case in particular for numbers $\beta$ having the finiteness property $(\mathrm{F})$ introduced in [17], which states that the set of non-negative real numbers with finite $\beta$-expansion coincides with the set of nonnegative elements of $\mathbb{Z}[1 / \beta]$ ). We deduce the following proposition from the results of $[17,1]$, stated in the framework of $\beta$-substitutions.

Proposition 47. Assume that $L=F\left(D_{\beta}\right)$, where $\beta>1$ is either 
- the positive root of the polynomial $X^{m}-t_{1} x^{m-1}-\cdots-t_{m}$, where $t_{i} \in \mathbb{Z}$, and $t_{1} \geq t_{2} \geq \cdots \geq t_{m}>0$

- the dominant root of the polynomial $X^{m}-t_{1} x^{m-1}-\cdots-t_{m}$, where $t_{i} \in \mathbb{N}$, and $t_{1}>\sum_{i=2}^{d}\left|t_{i}\right|>0$, and $\left(t_{1}, t_{2}\right) \neq(2,-1)$,

- a cubic Pisot unit.

Then the map $\varphi_{\mathbb{L}}$ is continuous and onto $\mathbb{T}^{m-1}$ ( $m=3$ in the third case); it is oneto-one except on a at most countable number of points; furthermore $\varphi_{\mathbb{L}}$ is a geometrical representation of $(\widetilde{\mathcal{K}}, \tau)$, the partition being given by the sets $\varphi_{\mathbb{L}}\{(x, y) \mid(x, y) \in$ $\left.\widetilde{\mathcal{K}}, y_{0}=q\right\}, q \in Q$.

Proof. The fact that $\varphi_{\mathbb{L}}$ is one-to-one except on a at most countable number of points comes from [17] for the first case, and from [1] for the last two points. It remains to prove that the sets $\varphi_{\mathbb{L}}\left\{(x, y) \mid(x, y) \in \widetilde{\mathcal{K}}, y_{0}=q\right\}, q \in Q$ are disjoint up to sets of zero Lebesgue measure. This is a direct consequence of the fact that $\beta$-substitutions satisfy the so-called strong coincidence condition, according to $[2]$.

\section{Some SPeCial CASES}

In [19], the odometer is defined on a set $\mathcal{R}$ of sequences of digits. Here, we have introduced an odometer on a set $\widetilde{\mathcal{K}}$ of pairs of infinite words. In this section, we show that in some particular situations, we can restrict ourselves to unidimensional sequences. So we exhibit hypothesis where the extra information given by the sequence of states is useless, or more precisely, the projection map $p_{1}: \widetilde{\mathcal{K}} \rightarrow \widetilde{\mathcal{L}}$ $(x, y) \mapsto x$ is injective, which implies, following Proposition 10, that both sets $\widetilde{\mathcal{K}}$ and $\widetilde{\mathcal{L}}$ are in one-to-one correspondence. The interest relies on the fact that the odometer can be directly defined on $\widetilde{\mathcal{L}}$.

Definition 48. Let $d \geq 1$. A regular language $L$ is said to be $d$-synchronizing if there exists a function $f: A^{d} \rightarrow Q$ such that for any word $w \in A^{*}$ of length $d$ and any $q \in Q, \delta(q, w)$ is equal to $f(w)$ (let us recall that $\delta$ denotes the transition function of the minimal automaton of $L)$. In other words, for any element $(x, y)=$ $\left(x_{0} x_{1} \ldots, y_{0} y_{1} \ldots\right)$ in $\widetilde{\mathcal{K}}$, for all $i \geq 0$ the state $y_{i}$ is completely determined by $x_{i} \cdots x_{i+d-1}$. A language is synchronizing if there exists a positive integer $d$ such that $L$ is $d$-synchronizing. Otherwise stated, this means that $y$ can be deduced from $x$.

Example 49. Consider the language accepted by the automaton $\mathcal{M}_{\sigma}$ depicted in Figure 4 of Example 35. Here, we represent in Figure 5 the automaton $\widehat{\mathcal{M}}_{\sigma}$. This

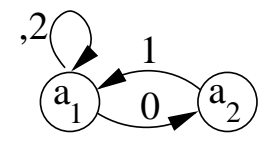

Figure 5. The automaton $\widetilde{\mathcal{M}_{\sigma}}$.

language is 1-synchronizing. Indeed, assume that $(x, y)$ is an element in $\widetilde{\mathcal{K}}$. The factors possibly appearing in $x$ are 00,01, 02, 10, 12, 20 and 22. Actually, 11 and 21 cannot occur in $x$ because no infinite path in the automaton depicted in Figure 5 contains such a factor. Clearly, if $x_{i} \in\{0,2\}$ then $y_{i}=a_{1}$ and if $x_{i}=1$ then $y_{i}=a_{2}$. 
Example 50. Continuing Examples 35 and 49, the language accepted by $\mathcal{M}_{\sigma}$ depicted in Figure 4 is 1-synchronizing and we have the function

$$
f: 0 \mapsto a_{1}, 1 \mapsto a_{2}, 2 \mapsto a_{1} .
$$

For this automaton $\mathcal{M}_{\sigma}$, we have $\delta\left(a_{1}, 0\right)=\delta\left(a_{2}, 0\right)=f(0)=a_{1}, \delta\left(a_{2}, 1\right)=f(1)=$ $a_{2}, \delta\left(a_{2}, 1\right)$ is the sink, $\delta\left(a_{1}, 2\right)=f(2)=a_{1}$ and $\delta\left(a_{2}, 2\right)$ is also the sink.

\section{REFERENCES}

[1] S. Akiyama, H. Rao, W. Steiner, A certain finiteness property of Pisot number systems, $J$. Number Theory, to appear.

[2] P. Arnoux, S. Ito, Pisot substitutions and Rauzy fractals, Bull. Belg. Math. Soc. Simon Stevin 8 (2001), 181-207.

[3] A. Bertrand-Mathis, Comment écrire les nombres entiers dans une base qui n'est pas entière, Acta Math. Acad. Sci. Hugar. 54 (1989), 237-241.

[4] V. Bruyère, G. Hansel, Bertrand numeration systems and recognizability, Latin American Theoretical INformatics (Valparaíso, 1995), Theoret. Comput. Sci. 181 (1997), 17-43.

[5] G. Barat, T. Downarowicz, P. Liardet, Dynamiques associées à une échelle de numération, Acta Arith. 103 (2002), 41-78.

[6] G. Barat, T. Downarowicz, A. Iwanik, P. Liardet, Propriétés topologiques et combinatoires des échelles de numération, Colloq. Math. 84/85 (2000), 285-306.

[7] H. Bruin, G. Heller, M. St. Pierre, Adding machines and wild attractors, Ergodic Theory Dynam. Systems., 17 (1997), 1267-1268.

[8] V. Canterini, A. Siegel, Automate des préfixes-suffixes associé à une substitution primitive, J. Théor. Nombres Bordeaux 13 (2001), 353-369.

[9] V. Canterini, A. Siegel, Geometric representation of substutions of Pisot type, Trans. Amer. Mah. Soc. 353 (2001), 5121-5144.

[10] J.-M. Dumont, A. Thomas, Systèmes de numération et fonctions fractales relatifs aux substitutions, Theoret. Comp. Sci. 65 (1989), 153-169.

[11] J.-M. Dumont, A. Thomas, Digital sum moments and substitutions, Acta Arith. 64 (1993), $205-225$.

[12] S. Eilenberg, Automata, Languages and Machines, Vol. A, Academic Press, New York, (1974).

[13] S. Fabre, Substitutions et $\beta$-systèmes de numération, Theoret. Comput. Sci. 137 (1995), 219-236.

[14] A. Fraenkel, Systems of numeration, Amer. Math. Monthly 92 (1985), 105-114.

[15] C. Frougny, On-line odometers for two-sided symbolic dynamical systems, Proceedings of DLT 2002, Lect. Notes in Comput. Sci. 2450 (2002), 405-416.

[16] C. Frougny, On the sequentiality of the successor function, Inform. and Comput. 139 (1997), $17-38$.

[17] C. Frougny and B. Solomyak, Finite beta-expansions, Ergodic Theory Dynam. Systems 12 (1992), 713-723.

[18] C. Frougny and B. Solomyak, On representation of integers in linear numeration systems, Ergodic theory of $Z_{d}$ actions (Warwick, 1993-1994), 345-368, London Math. Soc. Lecture Note Ser. 228, Cambridge Univ. Press, Cambridge, 1996.

[19] P. J. Grabner, P. Liardet, R. F. Tichy, Odometers and systems of numeration, Acta Arith. 70 (1995), 103-123.

[20] P. J. Grabner, M. Rigo, Additive functions with respect to numeration systems on regular languages, Monatsh. Math. 139 (2003), 205-219.

[21] M. Hollander, Greedy numeration systems and regularity, Theory Comput. Syst. 31 (1998), 111-133.

[22] P.B.A. Lecomte, M. Rigo, Numeration systems on a regular language, Theory Comput. Syst. 34 (2001), 27-44.

[23] P. Lecomte, M. Rigo, Real numbers having ultimately periodic representations in abstract numeration systems, to appear in Inform. and Comput. (2004).

[24] N. Loraud, $\beta$-shift, systèmes de numération et automates, J. Théor. Nombres Bordeaux 7 (1995), 473-498.

[25] M. Lothaire, Algebraic Combinatorics on words, Cambridge University Press, Cambridge, (2002).

[26] B. Mossé, Reconnaissabilité des substitutions et complexité des suites automatiques, Bull. Soc. Math. France 124 (1996), 329-346.

[27] G. Păun, A. Salomaa, Thin and slender languages, Discrete Appl. Math. 61 (1995), 257-270. 
[28] N. Pytheas Fogg, Substitutions in Dynamics, Arithmetics and Combinatorics, Lect. Notes in Math. 1794, Springer-Verlag, Berlin, (2002).

[29] M. Queffélec, LSubstitution dynamical systems. Spectral analysis, Lect. Notes in Math. 1294, Springer-Verlag, (1987).

[30] G. Rauzy, Sequences defined by iterated morphisms, Sequences (Naples/Positano, 1988), pp. 275-286, Springer, New York, 1990.

[31] M. Rigo, Numeration systems on a regular language: arithmetic operations, recognizability and formal power series, Theoret. Comput. Sci. 269 (2001), 469-498.

[32] M. Rigo, Construction of regular languages and recognizability of polynomials, Discrete Math. 254 (2002), 485-496.

[33] M. Rigo, A. Maes, More on generalized automatic sequences, J. Autom. Lang. Comb. 7 (2002), 351-376.

[34] M. Rigo, W. Steiner, Abstract $\beta$-expansions and ultimately periodic representations, prepublication.

[35] K. Schmidt, Algebraic coding of expansive group automorphisms and two-sided beta-shifts, Monatsh. Math. 129 (2000), 37-61.

[36] J. Shallit, Numeration systems, Linear recurrences, and Regular sets, Inform. and Comput. 113 (1994), 331-347.

[37] N. Sidorov, A. Vershik, Ergodic properties of the Erdös measure, the entropy of the golden shift, and related problems, Monatsh. Math. 126 (1998), 215-261.

[38] B. Solomyak, Substitutions, adic transformations and beta-expansions, Symbolic Dynamics and its applications, 135, P. Walters Editor (1992), 361-372.

[39] W. P. Thurston, Groups, tilings and finite state automata, Lectures notes distributed in conjunction with the Colloquium Series, in AMS Colloquium lectures, (1989).

[40] A. M. Vershik, Uniform algebraic approximation of shift and multiplication operators, Soviet Math. Dokl. 24 (1981), 97-100.

(V. Berthé)

LIRMM, Université MONTPELLIER II

161 RUE ADA

34392 Montpellier CedeX 5

FRANCE.

E-mail address: berthe@lirmm.fr

(M. Rigo)

UNIVERSITÉ DE LIÈGE

Institut DE MATHÉmatiQues

Grande Traverse 12 (B 37)

B-4000 LIÈGE

Belgium.

E-mail address: M.Rigo@ulg.ac.be 